\title{
Photorefractive quantum wells: transverse Franz-Keldysh geometry
}

\author{
Q. Wang, R. M. Brubaker, and D. D. Nolte \\ Department of Physics, Purdue University, West Lafayette, Indiana 47907 \\ M. R. Melloch \\ School of Electrical Engineering, Purdue University, West Lafayette, Indiana 47907
}

Received November 14, 1991; revised manuscript received March 19, 1992

\begin{abstract}
The photorefractive properties of semi-insulating AlGaAs-GaAs multiple quantum wells are described for the transverse Franz-Keldysh geometry with the electric field in the plane of the quantum wells. Combining the strong electroabsorption of quantum-confined excitons with the high resistivity of semi-insulating quantum wells yields large nonlinear optical sensitivities. The photorefractive quantum wells have effective nonlinear optical sensitivities of $n_{2} \approx 10^{3} \mathrm{~cm}^{2} / \mathrm{W}$ and $\alpha_{2} / \alpha_{0} \approx 10^{4} \mathrm{~cm}^{2} / \mathrm{W}$ for applied fields of $10 \mathrm{kV} / \mathrm{cm}$. Photorefractive gains approaching $1000 \mathrm{~cm}^{-1}$ have been observed in two-wave mixing under dc electric fields and stationary fringes. The transverse Franz-Keldysh geometry retains the general transport properties and behavior of conventional bulk photorefractive materials. The resonant excitation of free electrons and holes in the quantum wells leads to novel behavior associated with electron-hole competition. We demonstrate that under resonant excitation of electrons and holes the device resolution is fundamentally limited by diffusion lengths but is insensitive to long drift lengths.
\end{abstract}

\section{INTRODUCTION}

Photorefractive semiconductors have the advantage of large carrier mobilities and high speed, ${ }^{1,2}$ but they suffer from small linear electro-optic coefficients. Electro-optic properties are enhanced by using laser wavelengths close to band-gap absorption. The quadratic electro-optic effects associated with the fundamental absorption energy in semiconductors can be significantly larger than the linear electro-optic effect. The Franz-Keldysh effect ${ }^{3,4}$ is the change induced in the absorption near the band gap that is caused by an electric field. The field-induced absorption change is called electroabsorption..$^{5-9}$ Electrorefraction accompanies the electroabsorption ${ }^{10,11}$ and is related by causality through the Kramers-Kronig relation. 12,13 The Franz-Keldysh effect represents a resonant enhancement of the material electro-optic properties. The effect is strong only when the photon energy is close to the resonant transition energy from the valence band to the conduction band. However, significant electroabsorption can still occur for photon energies tuned to lower energies (within approximately $50 \mathrm{meV}$ ). Such near-resonant effects have been used for waveguide phase modulators ${ }^{14}$ and to enhance the photorefractive properties of bulk semiconductors. . $^{15,16}$

An alternative to using near-resonant frequencies with bulk excitons is to use the exciton wavelengths themselves in thin quantum-well structures. The quadratic electrooptic effects are strongest for photon energies tuned to sharp absorption features. For a resonant optical nonlinearity, the strength of the effect is proportional to the oscillator strength and varies inversely with the square of the transition linewidth. Strong and sharp exciton absorption features are therefore necessary to provide the largest possible electro-optic effect. Such sharp absorption features occur for quantum-confined excitons. ${ }^{17}$ Quantum-confined excitons are obtained by confining the exciton wave function within a thin quantum well. The quantum well consists of a semiconductor layer with quantum barriers formed from higher-band-gap materials. The band-edge discontinuity acts as a confinement potential that restricts the exciton wave function to the thin quantum well, effectively reducing the exciton to be quasi two dimensional. The binding energy of excitons in two dimensions is higher than in three because of the increased overlap of the electron and the hole wave functions with the attractive Coulomb potential. The higher binding energy for quantum-confined excitons produces two important effects: (1) It increases the oscillator strength of the exciton transition, and (2) it decreases the linewidth by increasing the lifetime of the exciton, thereby reducing the thermal ionization rate of the exciton into free electron-hole pairs. The larger oscillator strength and the smaller linewidth both contribute to significantly enhanced nonlinear optical and electro-optical properties, such as transverse quantum-confined exciton electroabsorption.

In this paper we describe the behavior of photorefractive quantum wells operating in the transverse Franz-Keldysh geometry. ${ }^{18}$ As for the common bulk photorefractive materials, high-sensitivity photorefractive performance depends on (1) large photoconductivity, (2) large dark resistivity, (3) large defect densities, and (4) large electrooptic effects. The photorefractive quantum wells satisfy all these requirements. In the transverse Franz-Keldysh geometry the electric field is applied in the plane of the quantum wells and is parallel to the grating vector written by intersecting laser beams. This geometry is identi- 
cal to the transport geometry commonly used for bulk photorefractive materials. However, the largest difference between the operation of a photorefractive quantumwell structure and nonresonant excitation in the bulk material is the direct generation of electron-hole pairs. The photoconductivity of the photorefractive quantumwell samples is caused not by photoionization of defects but by direct interband transitions. This difference raises interesting new behavior for the quantum-well samples that differ from nonresonant excitation and that have some features in common with resonant excitation of bulk materials. ${ }^{15}$

The structure of the quantum-well samples and the material processing are described in Section 2, including the electrical and the optical characterizations of the samples. The details of photorefractive transport under the conditions of electron-hole-pair production are presented in Section 3. The specifics of the transverse Franz-Keldysh geometry are addressed, and the onedimensional transport equations are solved. The respective roles of carrier diffusion and drift are described, with an emphasis on new behavior related to interband photoexcitation. Limitations of the one-dimensional transport model are discussed, with a list of possible consequences for vertical transport through or over the quantum barriers. Photorefractive four-wave mixing is described in Section 4, including degenerate and nondegenerate geometries with a description of high-order harmonic gratings formed by a combination of the quadratic electro-optic effect with transport nonlinearity. Two-wave mixing is described in Section 5, demonstrating energy transfer between two crossed beams in the photorefractive quantum wells. Photorefractive gains approaching $1000 \mathrm{~cm}^{-1}$ are observed under dc bias and stationary fringes. Speculations on the origins of the phase shift necessary for twowave mixing are offered, although the precise origin of the phase shift remains a mystery requiring further research. Finally, in Section 6 we discuss the ultrahigh sensitivity of the photorefractive quantum wells. A $10 \%$ transfer of energy and a saturation intensity of $10 \mu \mathrm{W} / \mathrm{cm}^{2}$ have been observed in two-wave mixing experiments.

\section{SEMI-INSULATING QUANTUM WELLS}

A. Sample Structure and Processing

All samples for this study were GaAs-AlGaAs multiplequantum-well structures grown by molecular-beam epitaxy in a Varian Gen-II chamber. We studied three samples. Two, labeled QW-1 and QW-2, were from the same growth. The third, RB-1, is from a different growth. As depicted in Fig. 1, each growth begins with a GaAs buffer layer grown on an undoped GaAs substrate. A 500-^ AlAs lift-off layer ${ }^{19}$ is grown next, followed by two stop-etch layers, one a $1000-\AA \mathrm{Al}_{0.5} \mathrm{Ga}_{0.5}$ As layer and the other a $7000-\AA \mathrm{Al}_{0.3} \mathrm{Ga}_{0.7} \mathrm{As}$ layer. The quantum wells are grown on the stop-etch layer, with 60 periods for each growth. For the QW-1 and QW-2 samples there are 75- $\AA$ GaAs wells and $100-\AA \mathrm{Al}_{0.3} \mathrm{Ga}_{0.7} \mathrm{As}$ barriers. The GaAs wells in the RB-1 sample are also $75 \AA$ wide, but the $\mathrm{Al}_{0.3} \mathrm{Ga}_{0.7} \mathrm{As}$ barriers are $150 \AA$ wide. On the wells is a 500 - $\AA$ layer of $\mathrm{Al}_{0.3} \mathrm{Ga}_{0.7} \mathrm{As}$ and, finally, a $50-\AA \mathrm{GaAs}$ cap to inhibit oxidation. After growth each 2-in.- (5-cm-) diameter wafer was cleaved into quarters.
For provision of deep-level traps the samples were proton implanted to provide implant damage ${ }^{0-23}$ A quarter wafer from each growth was first proton implanted from the quantum-well side with a dose of $10^{12} \mathrm{~cm}^{-2}$ at an energy of 135-160 keV. The penetration depth of the implant at these energies is 1.0 to $1.2 \mu \mathrm{m} .{ }^{24}$ The total depths of the multiple-quantum-well layers in Fig. 1 are 1.1 and $1.4 \mu \mathrm{m}$, respectively, for the two growths. To ensure that the samples are semi-insulating throughout, we removed the substrates, and the samples were implanted again from the etched side. Based on relativistic kinematics, approximately 40 atoms are displaced by each proton. ${ }^{25}$ However, some of the vacancy-interstice pairs recombine shortly after they are created. The volumedefect densities created by the implant are therefore estimated to be $N_{D} \approx 10^{17} \mathrm{~cm}^{-3}$. These defect densities are more than sufficient to compensate for the residual shallow-defect concentrations in the range of $N_{s} \approx 10^{15}$ $\mathrm{cm}^{-3}$ and to produce a semi-insulating material.

The GaAs substrate is opaque to the photon energies of interest close to the exciton transitions in the quantum wells. Our experiments are performed in a transmission geometry, requiring the substrates to be removed (in addition to requiring that the back sides of the samples be implanted). To remove the substrates, we cleaved $3 \mathrm{~mm} \times 3 \mathrm{~mm}$ samples from the quarter wafers. Different etching techniques were used to remove the substrate for each sample. For QW-1 and QW-2 each sample was epoxied to a glass slide from the quantum-well side, and then the substrate was lapped up to a thickness of $150 \mu \mathrm{m}$. For QW-1 the remaining portion of the substrate was removed by using a selective etching of $\mathrm{H}_{2} \mathrm{O}_{2} / \mathrm{NH}_{4} \mathrm{OH}$ at $\mathrm{pH} 7.0{ }^{26}$ For QW2 a different etching, using a volumetric 10:1 ratio of citric acid and $\mathrm{H}_{2} \mathrm{O}_{2}$ (30\%) (Ref. 27) was used, giving much better selectivity.

For RB-1 the multiple-quantum-well structure was separated from the substrate by dissolving the AlAs layer with a solution of $10 \% \mathrm{HF}$, which is referred to as the liftoff technique ${ }^{19}$ Before being etched, the sample top is coated with a waxlike material. During etching the wax coating bends the sample, allowing the etch to reach the edge of the AlAs layer and allowing the reactants to circulate away. The etching takes $\sim 24 \mathrm{~h}$ to separate a
QW-1 and QW-2

\begin{tabular}{|c|c|}
\hline & $50 \AA ̊$ GaAs \\
\hline \multicolumn{2}{|r|}{$500 \AA \mathrm{Al}_{0.3} \mathrm{Ga}_{0.7} \mathrm{As}$} \\
\hline \multirow{2}{*}{$60 \times\{$} & $75 \AA$ GaAs \\
\hline & $100 \AA \mathrm{Al}_{0.3} \mathrm{Ga}_{0.7} \mathrm{As}$ \\
\hline \multicolumn{2}{|r|}{$7,000 \AA \mathrm{Al}_{0.3} \mathrm{Ga}_{0.7} \mathrm{As}$} \\
\hline \multicolumn{2}{|r|}{$1,000 \AA A \mathrm{I}_{0.5} \mathrm{Ga}_{0.5}$ As } \\
\hline \multicolumn{2}{|r|}{$500 \AA ̊$ AlAs } \\
\hline \multicolumn{2}{|r|}{$0.75 \mu \mathrm{m}$ GaAs } \\
\hline Undop & d GaAs Substrate \\
\hline
\end{tabular}

RB-1

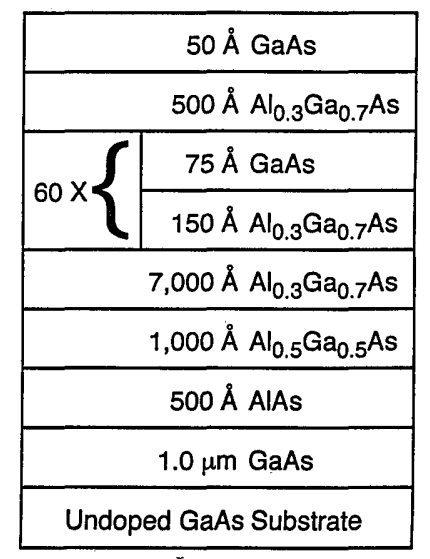

Fig. 1. Multiple-quantum-well structures used in the study. Sample RB-1 differs only in the thickness of the quantum barriers. 


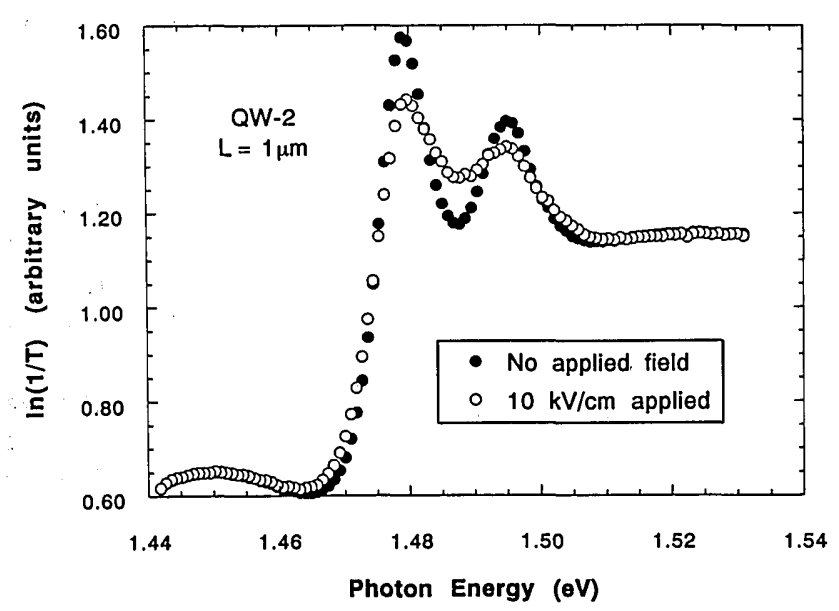

Fig. 2. Room-temperature absorption versus photon energy for sample QW-2 after sample processing. The quantum-confined heavy- and light-hole excitons are the dominant absorption features at 1.478 and $1.495 \mathrm{eV}$. Under an applied field of $10 \mathrm{kV} / \mathrm{cm}$ in the plane of the wells, the exciton line shapes broaden.

3-mm-square sample from the substrate. After liftoff the wax supports the fragile, $\sim 2-\mu \mathrm{m}$-thick sample. The sample is bonded to a glass slide by van der Waals bonding. ${ }^{28}$ After the bonding the wax coating is removed with a solvent, leaving a good optical surface. After the substrates were removed, gold electrodes were evaporated onto the samples $1 \mathrm{~mm}$ apart, and the samples were placed in optical mounts.

\section{B. Electrical and Optical Properties}

The resistivities of the samples after processing are typical of semi-insulating GaAs in the range of $5 \times 10^{7} \Omega \mathrm{cm}$. The high resistivity ensures that the proton implant has generated semi-insulating multiple quantum wells. The transmissions of the samples were measured as functions of photon energy, shown in Fig. 2 for sample QW-2. The quantum confinement of the excitons breaks the symmetry at the top of the valence band. Therefore the light hole and the heavy hole each have an absorption peak, as plotted in the figure. An estimate of $\alpha \approx 9 \times 10^{3} \mathrm{~cm}^{-1}$ for the peak absorption of the heavy-hole exciton can be made based on the thickness $L=1.04 \mu \mathrm{m}$ of the sample. There are weak Fabry-Perot fringes noticeable at energies above and below the excitons, which are caused by the sample thickness.

The photoconductivity was measured as a function of both applied voltage and incident intensity. As a function of applied voltage, the photoconductivity was linear (7 kV/cm was the highest applied field) for intensities of $0.3-300 \mathrm{~mW} / \mathrm{cm}^{2}$ at $\lambda=633 \mathrm{~nm}$ and $0.8-80 \mathrm{~mW} / \mathrm{cm}^{2}$ at $\lambda=843 \mathrm{~nm}$. The photoconductivity as a function of intensity was also measured at $\lambda=633 \mathrm{~nm}$ and $\lambda=843 \mathrm{~nm}$, with an applied field of $1 \mathrm{kV} / \mathrm{cm}$. The bipolar $\mu \tau$ products extracted from the curves are $3 \times 10^{-7} \mathrm{~cm}^{2} / \mathrm{V}$.

The linearity of the current-voltage plots of fields up to $7-\mathrm{kV} / \mathrm{cm}$ provides important evidence concerning the dominant photocarrier. In $\mathrm{GaAs}$, for fields above $4 \mathrm{kV} / \mathrm{cm}$, electrons gain sufficient energy to transfer from the direct band edge to the indirect band edge. The electron effective mass in the indirect band edge is significantly larger than the direct effective mass. Therefore the electron velocities saturate. This effect is well known ${ }^{29}$ and is the origin of Gunn oscillations ${ }^{30}$ in doped materials. The absence of saturated carrier drift velocities for fields up to $7 \mathrm{kV} / \mathrm{cm}$ indicates that the photoconductivity in the quantum wells is dominated by hole transport. Although interband transitions generate as many electrons as holes, the defect capture cross sections for electrons can be significantly larger than for holes. The short electron lifetime therefore removes electrons from the conduction band and allows holes to dominate the transport. Based on these arguments, and assuming a hole mobility of $400 \mathrm{~cm}^{2} / \mathrm{Vs}$, the hole lifetime from the measured $\mu \tau$ product is approximately $\tau_{h} \approx 1 \mathrm{~ns}$. These arguments are plausible and lead to a reasonable value for the hole lifetime.

\section{Transverse-Field Exciton Electroabsorption}

In bulk GaAs the binding energy of excitons is only $4 \mathrm{meV}$ at room temperature. The weakly bound exciton is easily ionized by thermal phonons, reducing the lifetime too much for detection of exciton peaks in the absorption. By confinement in a quantum well with a width smaller than the exciton's radius in the bulk, the binding energy of an exciton is increased to $10 \mathrm{meV}^{31}$ The higher binding energy results in clear excitonic absorption peaks, as shown in Fig. 2. The sharp peaks are caused by reduced thermal ionization and by an increase in the oscillator strength of the transition. As was mentioned above, confinement also breaks the symmetry between the light and the heavy holes, yielding both a heavy-hole and a light-hole exciton with different binding energies.

A field applied parallel to the plane of the quantum wells ionizes the excitons, resulting in lifetime broadening of the transition line shape. This broadening is the transverse quantum confined exciton electroabsorption, ${ }^{32}$ which is a special case of quantum-confined exciton electroabsorption. ${ }^{33,34}$ It does not depend on the sign of the applied field. Therefore, to lowest order, the broadening depends quadratically on the applied field. The broadening results in large changes in the absorption near the exciton peaks. The transmission for zero applied field and for an applied field of $10 \mathrm{kV} / \mathrm{cm}$ is shown in Fig. 2. The change in transmission was measured for each sample. The change in absorption was calculated with

$$
\begin{aligned}
\Delta \alpha(E) & =\frac{-1}{L} \ln \left(1+\frac{\Delta T}{T}\right), \\
\frac{\Delta T}{T} & =\frac{T(E)-T(0)}{T(0)}
\end{aligned}
$$

Reflection is assumed to be constant for the wavelengths of interest. Equation (2.1) is derived from the approximation $T \propto \exp (-\alpha L)$, which results in a less than $10 \%$ error in calculating the change in absorption, $\Delta \alpha$. A complete treatment involves modeling the sample as an asymmetric Fabry-Perot cavity with absorption. The transmission of such a cavity is described by

$$
T=\frac{n_{2}}{n_{0}} \frac{\left(1-R_{1}\right)\left(1-R_{2}\right) \exp (-\alpha L)}{\left[1-\left(R_{1} R_{2}\right)^{1 / 2} \exp (-\alpha L)\right]^{2}+4\left(R_{1} R_{2}\right)^{1 / 2} \exp (-\alpha L)(\sin \delta)^{2}},
$$




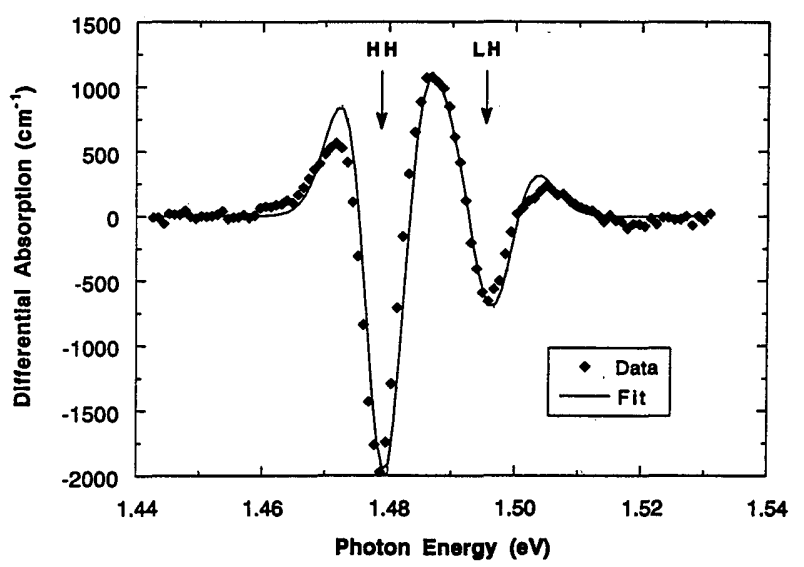

Fig. 3. Electroabsorption data plotted versus photon energy for an applied dc field of $10 \mathrm{kV} / \mathrm{cm}$. The solid curve is a fit of the data to Gaussian line shapes. The total oscillator strength is conserved in the Franz-Keldysh effect $(\mathrm{HH}$, heavy hole; $\mathrm{LH}$, light hole).

where

$$
\begin{aligned}
\delta & =(2 \pi / \lambda) n_{1} L, \\
R_{1} & =\frac{\left(n_{0}-n_{1}\right)^{2}+{k_{1}}^{2}}{\left(n_{0}+n_{1}\right)^{2}+{k_{1}}^{2}}, \\
R_{2} & =\frac{\left(n_{1}-n_{2}\right)^{2}+{k_{1}}^{2}}{\left(n_{1}+n_{2}\right)^{2}+{k_{1}}^{2}}, \\
k_{1} & =\alpha \lambda /(4 \pi),
\end{aligned}
$$

$n_{0}=1.5$ for glass, $n_{1} \approx 3.5$ for the semiconductor structure, and $n_{2}=1$ for air, yielding $R_{1}=0.16$ and $R_{2}=0.31$. We find that $9000 \mathrm{~cm}^{-1} \geq \alpha \geq 5000 \mathrm{~cm}^{-1}$ for wavelengths of interest. The maximum change in indices $(\Delta n)_{\max }=$ 0.01 and $(\Delta \alpha)_{\max }=2000 \mathrm{~cm}^{-1}$ implies that $(\Delta k)_{\max }=$ $\lambda(\Delta \alpha)_{\max } / 4 \pi=0.01$.

The reflectivities $R_{1}$ and $R_{2}$ change by less than $0.2 \%$ with the maximum changes in absorption and refractive index. Therefore the reflectivities are essentially constant for a given wavelength. The Fabry-Perot fringes measured below the band gap have a finesse $F=2$, which is consistent with the value calculated from

$$
F=\frac{\pi\left(R_{1} R_{2}\right)^{1 / 4}}{1-\left(R_{1} R_{2}\right)^{1 / 2}} .
$$

The low value of finesse makes $\Delta T$ insensitive to changes in the refractive index; $\Delta T$ varies by less than $1 \%$ with the change in index through the sin $\delta$ term. Therefore the changes in transmission depend only on absorption within a $1 \%$ error for calculating $\Delta T$. The dependence of $\Delta T$ on $\Delta \alpha$ is still governed by absorption and refractive index through the terms in the denominator of Eq. (2.2). To avoid the complex problem of accurately determining the absorption and refractive index, we drop the variation of the denominator, leading to at most a $10 \%$ error in calculating the change in absorption. The dominant change in transmission is through the exponential in the numerator, and the large value of absorption makes most terms in the denominator small compared with unity. The change in absorption is plotted in Fig. 3 for QW-2. The solid curve is the fit of the electroabsorption to Gaussian line shapes ${ }^{18}$ with a continuum contribution from absorption to free electron-hole pairs. The parameters of the fit are given in Table 1.

The change in absorption is accompanied by a change in the index of refraction, which may be calculated from the change in absorption with the Kramers-Kronig transformation:

$$
\Delta n(\lambda)=\frac{\lambda^{2}}{2 \pi^{2}} P \int_{0}^{\infty} \frac{\Delta \alpha\left(\lambda^{\prime}\right) \mathrm{d} \lambda^{\prime}}{\lambda^{2}-\lambda^{2}} .
$$

In Fig. 4, the calculated change in refractive index is shown with the absorption change. The quadratic dependence of the change in absorption and refractive index may be summarized with the coefficients $s_{1}$ and $s_{2}$, defined by

$$
\begin{aligned}
& \qquad \Delta \tilde{n}=(-1 / 2) n^{3} \tilde{s} E^{2}, \quad \tilde{s}=s_{1}+i s_{2}, \\
& \text { giving } \\
& \Delta n(E)=(-1 / 2) n^{3} s_{1} E^{2}, \quad \Delta \alpha(E)=(-2 \pi / \lambda) n^{3} s_{2} E^{2} .
\end{aligned}
$$

Coefficients $s_{1}$ and $s_{2}$ are plotted in Fig. 5 (here $n=3.5$ ).

\section{PHOTOREFRACTIVE TRANSPORT}

Photorefractive quantum wells operating in the transverse Franz-Keldysh geometry retain most of the basic features of photorefractive transport in bulk photorefractive materials. Diffusion and drift drive charge carriers from regions of high intensity to regions of low intensity. The carriers trap at defects, building up space-charge fields. These processes are described by one-dimensional transport equations, in which the photocarriers are assumed to remain in the quantum wells. Photorefractive quantum-well systems are typically operated at large applied electric fields and with interband transitions generating electrons and holes. These conditions can also be satisfied in bulk photorefractive materials. Therefore many of the conclusions of the transverse Franz-Keldysh geometry will be relevant for resonant and near-resonant ${ }^{15}$ operation of bulk photorefractive materials. However,

\begin{tabular}{|c|c|c|c|c|c|c|}
\hline Contribution & $\alpha\left(\mathrm{cm}^{-1}\right)$ & $\Omega(\mathrm{eV})$ & $\Gamma(\mathrm{meV})$ & $\Delta \alpha\left(\mathrm{cm}^{-1}\right)$ & $\Delta \Omega(\mathrm{eV})$ & $\Delta \Gamma(\mathrm{meV})$ \\
\hline Heavy hole & 7000 & 1.479 & 3.5 & -2000 & 0 & +1.4 \\
\hline Light hole & 4000 & 1.496 & 3.9 & -720 & 0 & +0.9 \\
\hline Continuum & 3000 & 1.490 & 6 & 0 & 0 & 0 \\
\hline
\end{tabular}
transport in photorefractive quantum wells can also include transport perpendicular to the quantum wells, such as tunneling into or thermal emission over the quantum barriers. A full description of the variety of effects that occur in photorefractive quantum wells will have to include this vertical transport in three-dimensional transport equations.

Table 1. Parameters for Fig. 3 


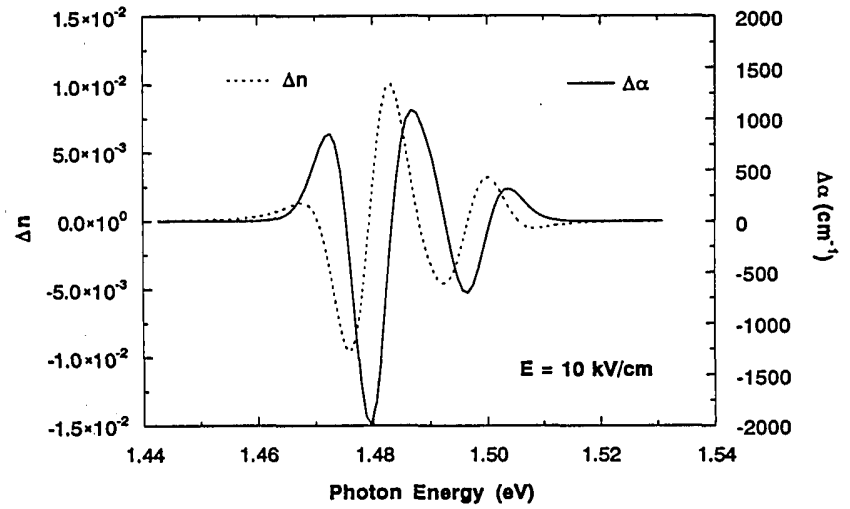

Fig. 4. Electroabsorption and electrorefraction for an applied field of $10 \mathrm{kV} / \mathrm{cm}$ versus photon energy.

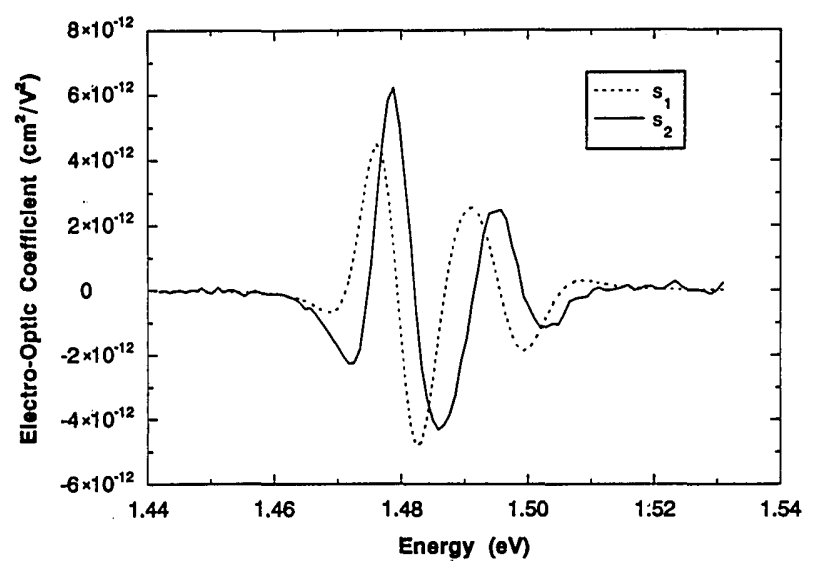

Fig. 5. Quadratic electro-optic coefficients $s_{1}$ and $s_{2}$ versus photon energy for quantum-confined excitons at room temperature.

\section{A. Transverse Franz-Keldysh Photorefractive Geometry}

The transverse Franz-Keldysh photorefractive geometry is shown in Fig. 6. The electric field is applied in the plane of the sample. Two coherent laser beams are incident upon the sample from the same side with external angles of incidences $\theta_{1}$ and $\theta_{2}$. The coherent interference between the two beams produces an interference fringe with a grating vector given by $\mathbf{K}_{g}=\mathbf{k}_{2}-\mathbf{k}_{1}$. When $\theta_{1}=\theta_{2}$, the grating vector $\mathbf{K}_{g}$ is in the plane of the sample. In the case of a thin grating only the component $K_{g x}=\mathbf{K}_{g} \cos \left(\theta_{2}-\theta_{1}\right)=K$, which lies in the plane, is important in defining transport properties and electrooptical gratings. However, for two-wave mixing, the grating vector $\mathbf{K}_{g}$ is important. Therefore, for two-wave mixing and related experiments, care must be exercized to maintain $\mathbf{K}_{g}$ in the plane of the layer. The parallel component of the grating vector is defined as $K=2 \pi / \Lambda$, where the fringe spacing $\Lambda$ is given by

$$
\Lambda=\lambda /\left(\sin \theta_{1}+\sin \theta_{2}\right) .
$$

The light intensity caused by the coherent interference between the two laser beams is

$$
I(x)=I_{0}(1+m \sin K x),
$$

where $m$ is the modulation index.

\section{B. One-Dimensional Transport Equations}

In semi-insulating multiple quantum wells operating in the transverse Franz-Keldysh geometry, the transport is predominantly in the direction of the grating vector. Therefore the equations that describe the photorefractive effect in semi-insulating multiple quantum wells are similar to the rate equations that describe the photorefractive process in bulk materials. ${ }^{35}$ If the photogenerated carriers are confined to the quantum wells, then one-dimensional transport equations should be a good approximation of the general photorefractive properties of the quantum wells. The transport equations include photocarrier generation, transport, trapping, and relaxation. These equations are

$$
\begin{aligned}
\frac{\partial n}{\partial t}-\nabla \frac{j_{e}}{e} & =I \alpha+\beta_{e} N_{D}^{0}-\sigma_{e} n v_{e} N_{D}^{+}-\gamma_{\mathrm{eh}} n p, \\
\frac{\partial p}{\partial t}+\nabla \frac{j_{h}}{e} & =I \alpha+\beta_{h} N_{D}^{+}-\sigma_{h} p v_{h} N_{D}^{0}-\gamma_{\mathrm{eh}} n p, \\
j_{e} & =e \mu_{e} n E+k_{B} T \mu_{e} \nabla n \\
j_{h} & =e \mu_{h} p E+k_{B} T \mu_{h} \nabla p \\
\frac{\partial}{\partial t}(n+ & \left.N_{A}^{-}-p-N_{D}^{+}\right)=\frac{\nabla}{e}\left(j_{e}+j_{h}\right), \\
\nabla E & =-\left(\frac{e}{\epsilon \epsilon_{0}}\right)\left(n+N_{A}^{-}-p-N_{D}^{+}\right),
\end{aligned}
$$

where $v_{e}$ and $v_{h}$ are the electron and the hole thermal velocities. Equations (3.3) and (3.4) are the generationrecombination equations. Equations (3.5) and (3.6) define the current densities. Equation (3.7) is the expression of charge conservation, and Eq. (3.8) is Gauss's law. In these equations $e$ and $h$ refer to electron properties and hole properties, respectively, where $\alpha$ is the absorption coefficient for electron-hole pair production, $I$ is the laser intensity, $j$ is the current density, $\beta$ is the carrier thermal emission rate, $\sigma$ is the defect capture cross section, and $\gamma_{\mathrm{eh}}$ is the electron-hole recombination cross section. The free-carrier concentrations are $n$ and $p$. The photocarrier generation rate $I \alpha$ is common to both the electron and the hole populations, as is the electron-hole recombination

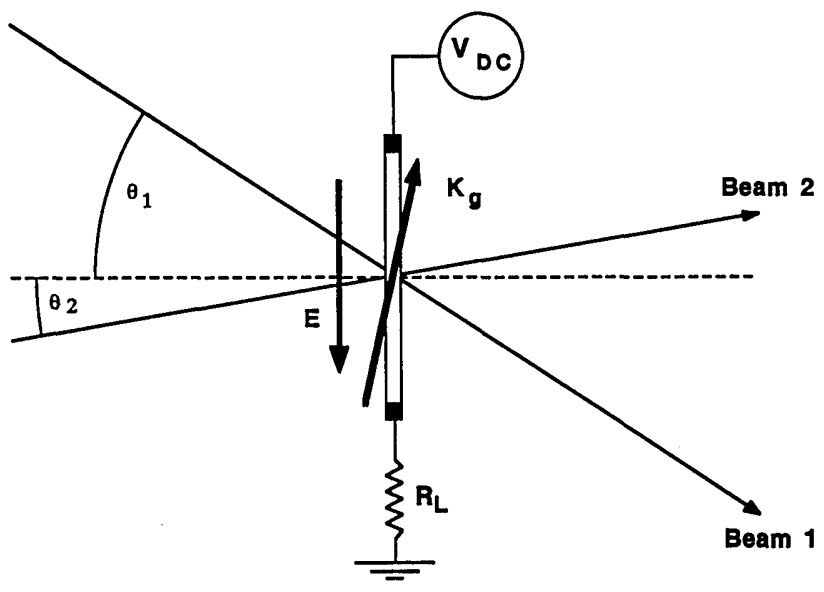

Fig. 6. Transverse Franz-Keldysh photorefractive geometry. For two-wave mixing in a thin grating, $\theta_{1}=\theta_{2}$, forcing $\mathbf{K}_{g}$ to lie in the plane of the sample. All measurements are performed under an applied electric field. 
rate $\gamma_{\mathrm{eh}} n p$. The recombination cross section $\gamma_{\mathrm{eh}}$ includes radiative recombination as well as nonradiative recombination through recombination defects. In the onedimensional approximation Eqs. (3.3)-(3.8) are identical to the photorefractive transport equations for resonant excitation of bulk materials.

For simplicity it is assumed that deep donors $N_{D}$ compensate for shallow acceptors $N_{A}$. The number of defects states is therefore

$$
N_{D}=N_{D}^{0}+N_{D}^{+} \text {. }
$$

In proton-implanted GaAs the exact mechanisms and the

The first-order terms are assumed to vary as $\exp (i K x)$, assuming steady state. The solution is expressed in terms of the following rates:

Dielectric relation rate,

Transition rate,

Ion recombination rate,

Drift rate,

Diffusion rate,

Direct recombination rate,

$$
\begin{aligned}
& \Gamma_{\mathrm{die}}=\mathrm{e} \mu_{\mathrm{e}} \mathrm{n}_{0} / \epsilon \epsilon_{0} \\
& \Gamma_{\mathrm{le}}=s_{e} I_{0}+\beta_{e}+\gamma_{e} n_{0} ; \\
& \Gamma_{\mathrm{Re}}=\gamma_{e}\left(N_{A}+n_{0}-p_{0}\right) \\
& \Gamma_{\mathrm{Ee}}=K \mu_{e} E_{0} ; \\
& \Gamma_{\mathrm{De}}=K^{2} k_{B} T \mu_{e} / e ; \\
& \Gamma_{\mathrm{eeh}}=\gamma_{\mathrm{eh}} \mathrm{p}_{0},
\end{aligned}
$$

with corresponding equations for holes. The rate equations are

$$
\left[\begin{array}{ccc}
-i \Gamma_{\mathrm{Ee}}+\Gamma_{\mathrm{De}}+\Gamma_{\mathrm{le}} & -\Gamma_{\mathrm{le}}+\Gamma_{\mathrm{ehh}} & -\Gamma_{\mathrm{die}}+\Gamma_{\mathrm{le}} \\
+\Gamma_{\mathrm{Re}}+\Gamma_{\mathrm{eeh}} & i \Gamma_{\mathrm{Eh}}+\Gamma_{\mathrm{Dh}}+\Gamma_{\mathrm{lh}} & \Gamma_{\mathrm{dih}}-\Gamma_{\mathrm{lh}} \\
-\Gamma_{\mathrm{lh}}+\Gamma_{\mathrm{eeh}} & +\Gamma_{\mathrm{Rh}}+\Gamma_{\mathrm{ehh}} & \\
\Gamma_{\mathrm{le}}+\Gamma_{\mathrm{Re}}+\Gamma_{\mathrm{lh}} & -\Gamma_{\mathrm{le}}-\Gamma_{\mathrm{lh}}-\Gamma_{\mathrm{Rh}} & \Gamma_{\mathrm{le}}+\Gamma_{\mathrm{lh}}
\end{array}\right]\left[\begin{array}{c} 
\\
n_{1} \\
p_{1} \\
A_{1}
\end{array}\right]=I_{1}\left[\begin{array}{c}
\alpha+s_{e}\left(N_{D}-N_{A}+p_{0}-n_{0}\right) \\
\alpha+s_{h}\left(N_{A}-p_{0}+n_{0}\right) \\
s_{e}\left(N_{D}-N_{A}+p_{0}-n_{0}\right)-s_{h}\left(N_{A}-p_{0}+n_{0}\right)
\end{array}\right]
$$

defects that make the material semi-insulating are unknown. The as-grown quantum-well materials are conducting and become semi-insulating after proton implantation. The radiation damage introduces deep defects that compensate for shallow dopants. For illustration purposes it is not important whether deep acceptors or deep donors are produced. The important features of photorefractive transport are contained within the assumption of a compensating defect that pins the Fermi level and traps photocarriers.

The zero-order solutions for $n_{0}$ and $p_{0}$ in Eqs. (3.3)-(3.9) are solved by assuming homogeneous illumination $I_{0}$. These solutions are

$$
\begin{aligned}
& n_{0}=\frac{I_{0} \alpha+I_{0} s_{e}\left(N_{D}-N_{A}-n_{0}+p_{0}\right)+\beta_{e}}{\gamma_{e}\left(N_{A}+n_{0}-p_{0}\right)+\gamma_{e h} p_{0}}, \\
& p_{0}=\frac{I_{0} \alpha+I_{0} s_{h}\left(N_{A}+n_{0}-p_{0}\right)+\beta_{h}}{\gamma_{h}\left(N_{D}-N_{A}-n_{0}+p_{0}\right)+\gamma_{e h} n_{0}}
\end{aligned}
$$

where $\alpha$ is the absorption coefficient for the generation of electron-hole pairs. The values for $\alpha$ are in the range of $10^{4} \mathrm{~cm}^{-1}$, compared with typical absorption coefficients of $\sim 1 \mathrm{~cm}^{-1}$ for deep defects. The electron-hole pair production rate is much larger than photoionization rates from the deep-level defects. Photogeneration across the band gap will therefore dominate the transport properties. The values of $n_{0}$ and $p_{0}$ on the right-hand sides of Eqs. (3.10) and (3.11) can be neglected under weak illumination. Laser intensities of $1 \mathrm{~mW} / \mathrm{cm}^{2}$ will generate freecarrier concentrations in the range $10^{10}-10^{12} \mathrm{~cm}^{-3}$, which are typically much smaller than $N_{A}$ or $N_{D}$.

The first-order solutions of Eqs. (3.3)-(3.9) are obtained in the small-modulation-amplitude approximation. ${ }^{35}$ The parameters can be expressed as $n=n_{0}+n_{1}, p=p_{0}+$ $p_{1}$, and $E=E_{0}+E_{1}$, where $E_{0}$ is the applied electric field. where $A_{1}=\epsilon_{0} \epsilon i K E_{1} / e$ is the space-charge density. These equations are similar to those for nonresonant bulk photorefractive materials. In the case of resonant excitation the extra terms that arise represent direct excitation of electron-hole pairs and recombination of the pairs.

The solutions of $E_{1}$ from Eq. (3.12) are shown in Fig. 7 as functions of fringe spacing for different applied fields $E_{0}$. The defect density is $N_{D}=1 \times 10^{17} \mathrm{~cm}^{-3}$, with a compensation ratio $r=N_{A} / N_{D}=0.9$ and other parameters as given in Table 2. These parameters are chosen to

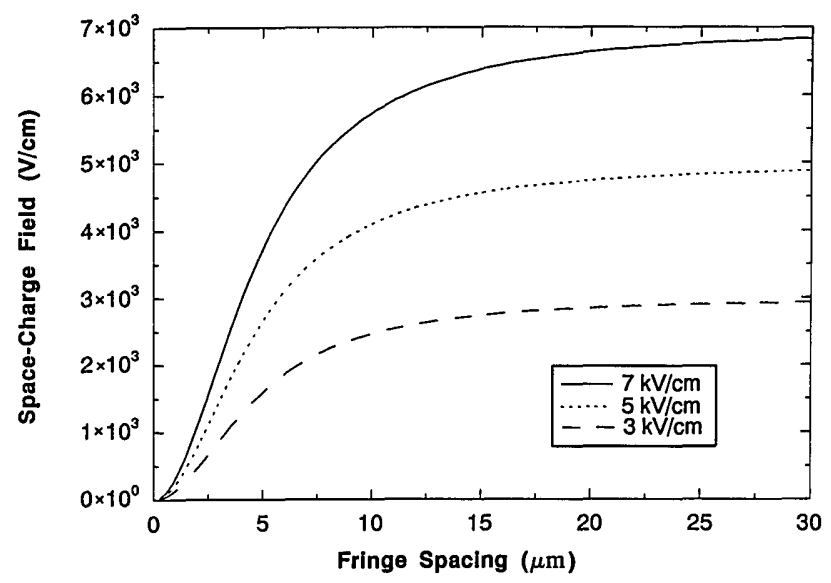

Fig. 7. Space-charge field amplitude versus fringe spacing for varying applied fields from numerical simulations of the quantum wells.

Table 2. Parameters for Fig. 7 


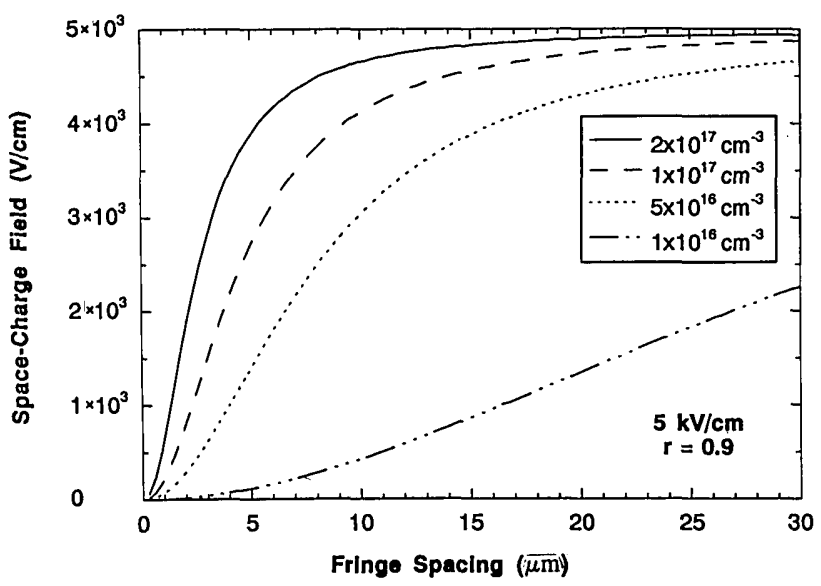

Fig. 8. Space-charge field amplitude versus fringe spacing for varying defect concentrations from numerical simulations of the quantum wells. The compensation ratio is $r=0.9$, with an applied field of $5 \mathrm{kV} / \mathrm{cm}$. The diffusion length determines the device resolution.

be representative but are not supposed to represent the exact values of the photorefractive quantum wells. The space-charge field drops quickly for fringe spacings below $10 \mu \mathrm{m}$. With an effective defect density of $(1-r) r N_{D}=$ $10^{16} \mathrm{~cm}^{-3}$ and an applied field of $5 \mathrm{kV} / \mathrm{cm}$, the material
On the other hand, the characteristic length is strongly dependent on defect concentration. The space-charge fields, for an applied field of $5 \mathrm{kV} / \mathrm{cm}$, are shown in Fig. 8 as functions of fringe spacing for different defect densities. Defect densities have a direct effect on transport lengths because of the dependence of carrier lifetime on defect density. Lower defect densities lead to larger characteristic lengths $\Lambda_{c}$ because the carrier lifetime is increased. This characteristic length defines the resolution of a photorefractive device and is therefore an important feature of photorefractive quantum wells.

The origin of the characteristic length can be understood by considering the transition rates in Eq. (3.12). These rates are shown in Fig. 9 for the material parameters given in Table 2. The curves are functions of fringe spacing for an electric field of $5 \mathrm{kV} / \mathrm{cm}$. Several of the rates, such as the transition rates $\Gamma_{l e}, \Gamma_{\mathrm{lh}}$, the dielectric relaxation rates $\Gamma_{\text {die }}, \Gamma_{\text {dih }}$, and the direct recombination rates $\Gamma_{\text {eeh }}, \Gamma_{\text {ehh }}$, are negligibly small. The transition rates are small because of the large absorption across the band gap. The dielectric relaxation rates are also small under conditions of weak illumination but in addition require that there be sufficient trap density to support the space charge (i.e., the system is not trap limited). By keeping only the largest terms in the solution to Eq. (3.12), we find that the solution for the space-charge density is

$$
A_{1}=-I_{0} \alpha\left[\frac{-\Gamma_{\mathrm{Rh}}\left(-i \Gamma_{\mathrm{Ee}}+\Gamma_{\mathrm{De}}\right)+\Gamma_{\mathrm{Re}}\left(i \Gamma_{\mathrm{Eh}}+\Gamma_{\mathrm{Dh}}\right)}{\Gamma_{\mathrm{dih}} \Gamma_{\mathrm{Rh}}\left(-i \Gamma_{\mathrm{Ee}}+\Gamma_{\mathrm{De}}+\Gamma_{\mathrm{Re}}\right)+\Gamma_{\mathrm{die}} \Gamma_{\mathrm{Re}}\left(i \Gamma_{\mathrm{Eh}}+\Gamma_{\mathrm{Dh}}+\Gamma_{\mathrm{Rh}}\right.}\right]
$$

becomes trap limited only for fringe spacings smaller than $0.2 \mu \mathrm{m}$. Therefore this dropoff is not caused by trap limitation. Furthermore, the characteristic length in Fig. 7 is approximately $\Lambda_{c}=5 \mu \mathrm{m}$ for each of the curves, independent of applied field. The characteristic length $\Lambda_{c}$ is therefore not dependent on the drift length in the material.
This solution is expressed in terms of the space-charge field as

$$
E_{1}=-E_{0}\left[\frac{K L_{\mathrm{Ee}}+i K^{2} L_{\mathrm{De}}{ }^{2}+K L_{\mathrm{Eh}}+i K^{2} L_{\mathrm{Dh}}^{2}}{L_{\mathrm{Eh}}\left(K^{2} L_{\mathrm{De}}{ }^{2}+1\right)+L_{\mathrm{Ee}}\left(K^{2} L_{\mathrm{Dh}}{ }^{2}+1\right)}\right]
$$

where the drift and the diffusion lengths are given by

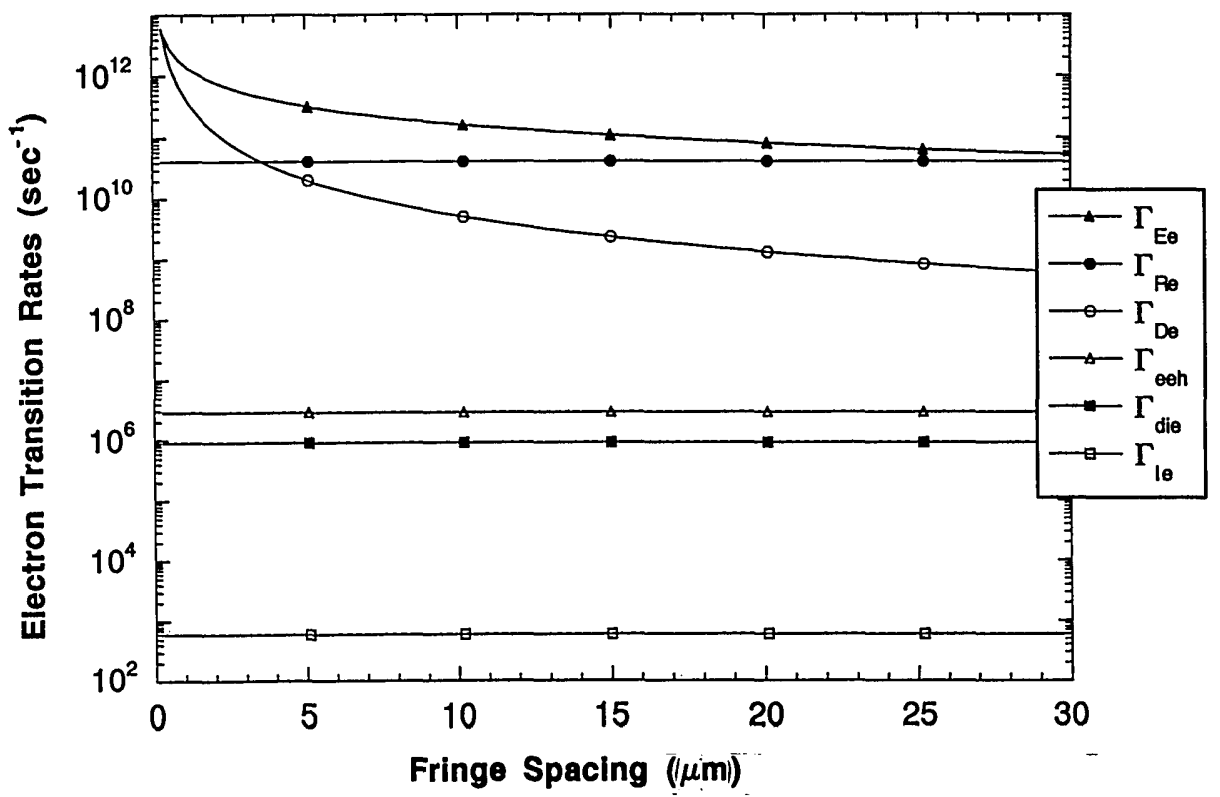

Fig. 9. Electron transition rates versus fringe spacing from numerical simulations. The rates $\Gamma_{\text {le }}, \Gamma_{\text {die, }}$ and $\Gamma_{\text {eeh }}$ are all negligible compared with the drift, diffusion, and recombination rates. Similar results apply for holes. 


$$
L_{E}=\mu \tau E, \quad L_{D}^{2}=\mu \tau k_{B} T / e .
$$

The expression for the space-charge field can be written directly in terms of the applied field $E_{0}$ and the diffusion field $E_{D}$ as

$$
E_{1}=-\frac{1}{\mu_{h} \tau_{h} \mu_{e} \tau_{e} K}\left\{\frac{\mu_{e} \tau_{e}\left(E_{0}+i E_{D}\right)+\mu_{h} \tau_{h}\left(E_{0}-i E_{D}\right)}{2 E_{D}+\left[1 /\left(\mu_{e} \tau_{e} K\right)\right]+\left[1 /\left(\mu_{h} \tau_{h} K\right)\right]}\right\},
$$

where $E_{D}=k_{B} T K / e$. In Eq. (3.16) the role of the $\mu \tau$ product for electrons and holes can be seen explicitly. The most important feature of Eq. (3.16) is the diffusion field $E_{D}$ in the denominator. In spite of the fact that $E_{0} \gg E_{D}$, diffusion plays a crucial role in limiting spacecharge fields under resonant excitation.

The characteristic length observed in Figs. 7 and 8 is given by

$$
K_{c}^{2}\left\langle L_{D}\right\rangle^{2}=1,
$$

where the bipolar diffusion length is given by

$$
\left\langle L_{D}\right\rangle^{2}=\left(k_{B} T / e\right)\langle\mu \tau\rangle,
$$

with the bipolar $\mu \tau$ product defined by

$$
\langle\mu \tau\rangle=\frac{1}{1 / 2\left[\left(1 / \mu_{e} \tau_{e}\right)+\left(1 / \mu_{h} \tau_{h}\right)\right]} .
$$

The resolution of the photorefractive quantum wells operating in the transverse Franz-Keldysh geometry is therefore limited by the bipolar diffusion length. This limitation is easily addressed by using higher protonimplant doses to reduce the diffusion length and to achieve better device resolution.

\section{Three-Dimensional Transport}

Equation (3.12) and the following equations are all based on one-dimensional transport. For the one-dimensional equations to be relevant, the material must be homogeneous. While this assumption is valid for thin bulk materials, it represents an approximation for the quantum-well samples, in which the carriers are assumed to be perfectly confined within the quantum wells. In fact the carrier wave functions penetrate the quantum barriers, allowing free carriers in the wells to tunnel into defects in the barriers. Phonon-assisted tunneling allows the carriers to tunnel even further, because phonons can promote the carriers into excited states of the quantum wells that are more weakly confined. Finally, thermionic emission of carriers out of the wells and over the barriers occurs. Each of these processes represents vertical transport, transport of charge along the growth direction of the multilayer structure. These processes are illustrated in Fig. 10.

The situation becomes even more complicated when carrier heating is included. The electric fields applied in the transverse Franz-Keldysh geometry range to $10^{4} \mathrm{~V} / \mathrm{cm}$ and above. At these field strengths the carrier gas in the wells can attain a significantly higher temperature than the lattice temperature. Electron temperatures of $500 \mathrm{~K}$ are characteristic of fields of this strength. At these temperatures thermionic emission over the quantum barriers becomes the dominant vertical transport mechanism. It should also be noted that the hot carriers will also influence diffusion lengths, which were shown to be particularly important through Eq. (3.14).

It is beyond the scope of this paper to include threedimensional transport explicitly. Many of the features that we observe in the photorefractive quantum wells can be described well by the one-dimensional transport equations. However, there are other features that defy explanation through Eq. (3.12). Examples include differences between degenerate and nondegenerate four-wave mixing and the origin of the phase shift observed in two-wave mixing. In order to explain these more complicated effects, it is probable that a complete three-dimensional transport solution will be necessary.

\section{FOUR-WAVE MIXING}

\section{A. Diffraction from Thin Gratings}

\section{Raman-Nath Diffraction}

For a three-dimensional periodic elastic scatterer, in which the light wave does not change its frequency through the interaction, the direction of the diffraction beam is given by the Bragg condition. The wave-vector difference $\Delta k$ between the incident and the diffracted waves must equal the grating vector. Consider a slab with a complex refractive-index distribution

$$
n_{p}=\left\{\begin{array}{cl}
n-\Delta n^{\prime} \cos (K x+\phi) & \text { for } 0<\mathrm{y}<\mathrm{L} \\
1 & \text { otherwise }
\end{array},\right.
$$

where $n$ and $\Delta n^{\prime}=\Delta n+i \Delta \alpha / 2 k$ are complex numbers. For large $L$ the wave-vector change $\Delta k$ must equal a multiple of the grating vector $K$. However, the situation changes in the thin-grating limit because of the finite linewidth of the spatial frequency spectrum of $n^{\prime}$ in the $y$ direction. As a result, a small $y$ component of $\Delta k$ can be tolerated, i.e., Raman-Nath diffraction. If we denote the $y$ component of $\Delta k$ to be $\delta k$, the intensity of the diffrac-

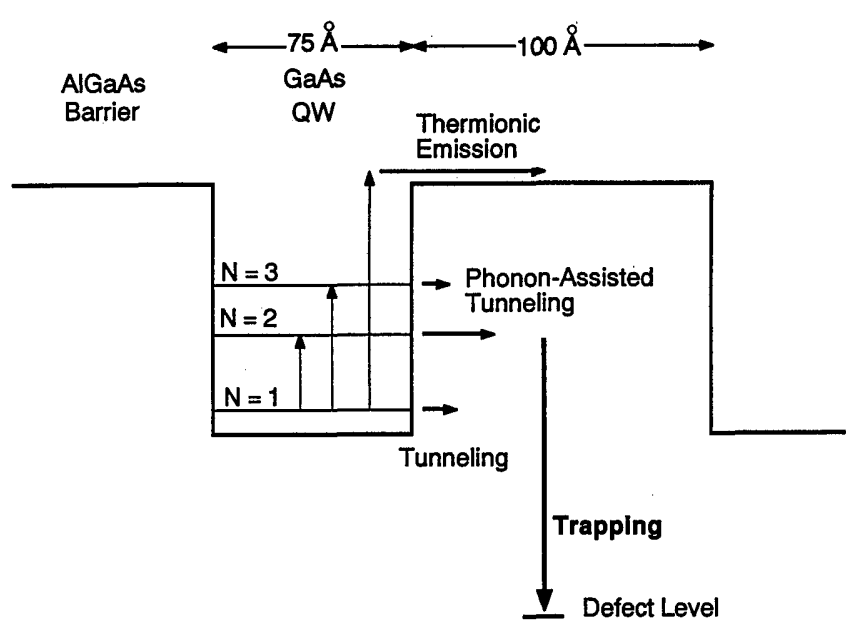

Fig. 10. Vertical transport processes. Carriers can escape the quantum wells by tunneling, phonon-assisted tunneling, and thermionic emission. The space-charge trapped at defects in the barriers is expected to play a role in photorefractive properties. 
Ar-Ion laser

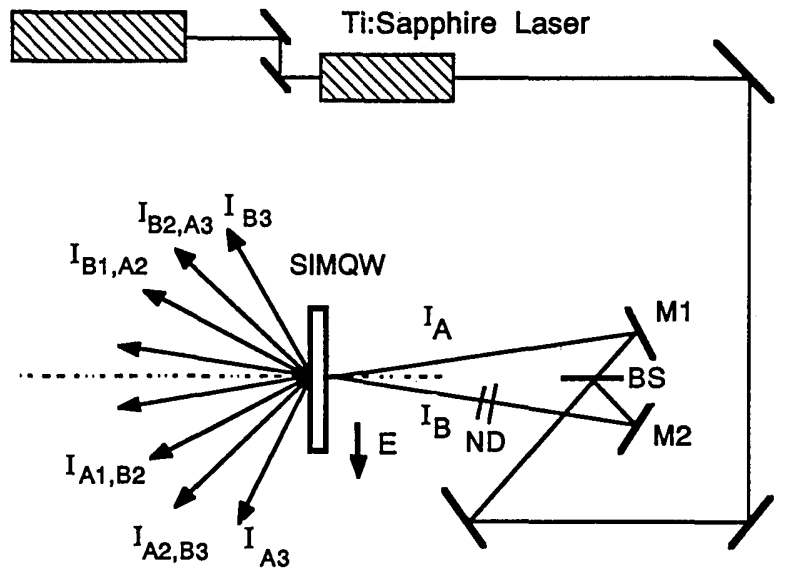

Fig. 11. First-order diffraction from high spatial harmonic gratings in the semi-insulating multiple quantum wells (SIMQW): M's, mirrors; BS, beam splitter; ND, neutral-density filter.

tion beam is proportional to

$$
\left|\int_{0}^{L} \mathrm{~d} y \exp (i \delta k y)\right|^{2}=\left|\frac{\exp (i \delta k L)-1}{\delta k}\right|^{2} .
$$

When the incident beam is almost perpendicular to the slab and the imaginary part of $n$ is negligibly small, Eq. (4.2) can be estimated as

$$
\delta k=K^{2} /(2 n k),
$$

where $k$ is the wave number of the incident wave.

The Raman-Nath and Bragg regimes are distinguished by a dimensionless parameter $Q$, where $Q<1$ for the Raman-Nath regime and $Q>1$ for the Bragg regime. ${ }^{36,37}$ The parameter $Q$ is defined by

$$
Q=4 \delta k L=2 \pi L \lambda /\left(n \Lambda^{2}\right),
$$

where $\Lambda$ is the periodicity of the grating. In our measurements $L=1 \mu \mathrm{m}, \lambda=840 \mathrm{~nm}$, and $n=3.6$. For GaAs multiple quantum wells, the parameter is

$$
Q=2.93\left(\mu \mathrm{m}^{2}\right) / \Lambda^{2},
$$

which places the experimental conditions in the strong Raman-Nath regime for $\Lambda>3 \mu \mathrm{m}$. According to Eq. (4.2) the intensity of the diffraction beam depends on $Q$ as

$$
\frac{\sin (Q / 8)^{2}}{(Q / 8)^{2}}
$$

In the Raman-Nath regime various orders of diffraction can be observed simultaneously. For an incident beam with an incident angle $\theta_{\text {in }}$, shown in Fig. 11, the direction of the diffraction beam for the $m$ th order is given by

$$
\sin \theta_{\text {out }}=\sin \theta_{\text {in }}+m \mathrm{~K} \lambda / 2 \pi \text {. }
$$

\section{Diffraction Efficiency}

In the Raman-Nath regime high-order diffraction is possible. A simple method for calculating the diffraction efficiency is based on the concept of wave-front modulation. Consider a thin grating in which the refractive index is given by Eq. (4.1). For an incident plane wave the transmitted wave for $\Delta n^{\prime}=0$ is represented by $E=$ $E_{t} \exp \left[i\left(k_{1} x+k_{2} y\right)\right]$, which is taken as the zero-order approximation in our calculation. The spatial modulation of the complex refraction index will modify the wave front of the transmission wave by a complex phase shift $\delta$, which is given by Eq. (4.8) in the first-order approximation:

$$
\delta=\left(k \Delta n L / \cos \theta^{\prime}+i \Delta \alpha L / 2 \cos \theta^{\prime}\right),
$$

where $\theta^{\prime}=\tan ^{-1}\left(k_{y} / k_{x}\right)$ and $k_{x}$ and $k_{y}$ are the $x$ and $y$ components of the wave vector in the slab. The modified transmitted wave becomes

$$
E=E_{t} \exp \left[i\left(k_{1} x+k_{2} y-\delta\right)\right] .
$$

With the Bessel-function identity

$$
\exp [i \delta \cos (x+\phi)]=\sum_{-\infty}^{\infty} J_{m}(\delta) \exp [-i m(x+\phi-\pi / 2)]
$$

the transmitted field can be written as

$$
\begin{aligned}
E= & E_{t} \sum_{-\infty}^{\infty} J_{m}(-\delta) \exp \left\{i \left[\left(k_{1}-m K\right)_{x}+k_{2} y\right.\right. \\
& -m(\phi-\pi / 2)]\} .
\end{aligned}
$$

The diffraction efficiency for $m$ th-order diffraction is given by

$$
\eta_{m}=\left|J_{m}^{2}(\delta)\right|=J_{m}^{2}\left(\left|\frac{2 \pi \Delta n L}{\lambda \cos \theta^{\prime}}+i \frac{\Delta \alpha L}{2 \cos \theta^{\prime}}\right|\right) .
$$

When the spatial modulation of the refractive index is small, the above expression can be simplified by using

$$
J_{m}(x) \approx x^{m} /\left(2^{m} m !\right)
$$

for small $x$, where $m$ is any nonnegative integer. This yields

$$
\eta_{m}=\frac{1}{2^{2 m} m !^{2}}\left[\left(\frac{2 \pi \Delta n L}{\lambda \cos \theta^{\prime}}\right)^{2}+\left(\frac{\Delta \alpha L}{2 \cos \theta^{\prime}}\right)^{2}\right]^{m} .
$$

\section{Absorption and Index Gratings}

In photorefractive multiwave mixing a spatial modulation of the absorption coefficient and refractive index is established in semi-insulating multiple quantum wells through transverse quantum-confined exciton electroabsorption. This grating is composed of high-order harmonic components because of two different nonlinearities: (1) the quadratic electro-optic effect and (2) the nonlinearities in the transport equations (3.3)-(3.6). The internal field is represented by ${ }^{37}$

$$
\begin{aligned}
E= & E_{0}+E_{\mathrm{sc}} \\
= & E_{0}-\left[E_{1} \cos \left(K x+\phi_{1}\right)+E_{2} \cos \left(2 K x+\phi_{2}\right)\right. \\
& \left.+E_{3} \cos \left(3 K x+\phi_{3}\right)\right],
\end{aligned}
$$

where $E_{0}$ is the applied field and $E_{1}, E_{2}$, and $E_{3}$ are the first-, the second-, and the third-harmonic components, respectively, of the screening field. We neglect harmonics higher than the third, because the amplitudes decrease rapidly with increasing order. The Fourier analysis of the 
photorefractive grating is made by considering $E^{2}$ :

$$
\begin{aligned}
E_{\mathrm{sc}}^{2}= & {\left[E_{0}^{2}+1 / 2\left(E_{1}^{2}+E_{2}^{2}+E_{3}^{2}\right)\right] } \\
& +\left[-2 E_{0} E_{1} \cos \left(K x+\phi_{1}\right)\right. \\
& +E_{1} E_{2} \cos \left(K x+\phi_{2}-\phi_{1}\right) \\
& \left.+E_{2} E_{3} \cos \left(K x+\phi_{3}-\phi_{2}\right)\right] \\
& +\left[1 \frac{1}{2} E_{1}^{2} \cos \left(2 K x+2 \phi_{1}\right)\right. \\
& -2 E_{0} E_{2} \cos \left(2 K x+\phi_{2}\right) \\
& \left.+E_{1} E_{3} \cos \left(2 K x+\phi_{3}-\phi_{1}\right)\right] \\
& +\left[-2 E_{0} E_{3} \cos \left(3 K x+\phi_{3}\right)\right. \\
& \left.+E_{1} E_{2} \cos \left(3 K x+\phi_{1}+\phi_{2}\right)\right] .
\end{aligned}
$$

The term in the first set of square brackets represents the average squared field. The term in the second set of square brackets represents the first-harmonic grating that is generated by mixing the first harmonic with the dc field, by mixing the second-harmonic electric field with the first harmonic, and by mixing the third harmonic with the second. The term in the third set of brackets represents the second-harmonic grating that is generated by the square of the first-harmonic field with itself, by mixing the second-harmonic electric field with the dc field, and by mixing the third harmonic with the first. Finally, the term in the last set of brackets represents the third-harmonic grating, which cannot exist without the transport nonlinearity.

Several kinds of wave-mixing experiments are dicussed in Subsection 4.B, each of them exploring a different aspect of the photorefractive semi-insulating multiple quantum wells. Two-wave mixing arises from the firstharmonic term of Eq. (4.15), including a phase shift to allow for photorefractive gain. Four-wave mixing is also related to the amplitude of the first-harmonic grating. Diffraction from the higher harmonics, corresponding to the third and fourth sets of brackets of Eq. (4.15), is also observable.

\section{B. Degenerate Four-Wave Mixing}

Details of degenerate four-wave mixing have been given elsewhere $^{18}$ and are similar to the behavior of nondegenerate four-wave mixing, which is described in Subsection 4.C. Therefore we highlight principally the features of high-order spatial harmonic gratings in this section.

High-order spatial harmonic gratings can be generated in semi-insulating multiple quantum wells by two processes: (1) the nonlinearity of photorefractive transport and (2) the quadratic electro-optic effect. Photorefractive transport in semi-insulating multiple quantum wells is described by a set of nonlinear equations. Generally, the solution is composed of various harmonic components for a pump-light intensity $I(x)=I_{0}(1+m \cos K x)$. Firstorder diffraction from the first-, the second-, and the thirdharmonic gratings is observed in the semi-insulating quantum wells when the modulation index $m$ approaches 1 . The possibility of observing second- or higher-order diffractions can be ruled out, because a maximum diffraction efficiency of $10^{-8}$ for the second-order diffraction is expected, which is below our detectability.
Before describing the experimental details, we consider the relationship between the modulation index $m$ and the second-harmonic component of the screening field. For the sake of simplicity, consider the origin of $E_{2}$ by assuming that there is only one kind of dominant photocarrier responsible for the photorefractive transport. In the steady state the current continuity equation is given by

$$
\mu \nabla(n E)=\gamma I_{0}[1+m \cos (K x)]-\left(n-n_{d}\right) / \tau,
$$

where $\mu$ is the mobility, $n$ is the carrier density, $n_{d}$ is the dark carrier density, $\gamma$ is the carrier-generation rate, and $\tau$ is the lifetime of a free carrier. We make a Fourier analysis of $n$, giving solutions $n=n_{0}+2 \operatorname{Re}\left[n_{1} \exp (i k x)\right]$ and $E=E_{0}+2 \operatorname{Re}\left[E_{1} \exp (i k x)+E_{2} \exp (i 2 k x)\right]$. Substituting these solutions into Eq. (4.16) yields

$$
E_{2} / E_{1}=-n_{1} / n_{0} \text {. }
$$

For $m \ll 1$, both $n_{1}$ and $E_{1}$ are proportional ${ }^{38}$ to $m$, which yields $E_{2}$ proportional to $m^{2}$. Considering Eqs. (4.13) and (4.15) for the first-order diffraction, a diffraction efficiency proportional to $\mathrm{m}^{4}$ for a double spatial frequency grating and to $m^{2}$ for a fundamental spatial frequency grating is expected. Therefore, in order to observe the effect of the high-harmonic gratings, one must violate the conditions for the small-modulation-amplitude approximation.

The experimental setup for multiwave mixing is shown in Fig. 11. A modulated dc field is applied across the semi-insulating multiple quantum wells. The photon energy dependence of the diffraction efficiencies for sample QW-1 was measured for a fringe spacing of $8.8 \mu \mathrm{m}$. The result is shown in Fig. 12. Taking the largest terms in Eq. (4.15), we can estimate $E_{1} / E_{2}$. As a result we have

$$
\frac{\eta_{1}(2 K)}{\eta_{1}(K)}=\left(\frac{E_{2}}{E_{1}}-\frac{E_{1}}{4 E_{0}}\right)^{2} \approx \frac{1}{4},
$$

which yields $E_{2} / E_{1}=75 \%$. This value is consistent with numerical results from Ref. 39 for a large modulation depth and an applied field close to the trap-limited value.

The dependence of the diffraction signal from the first and the second spatial harmonic gratings on the modulation index $m$ was measured at the zero crossings of electroabsorption for $\Lambda=4.6 \mu \mathrm{m}$. The results are shown in

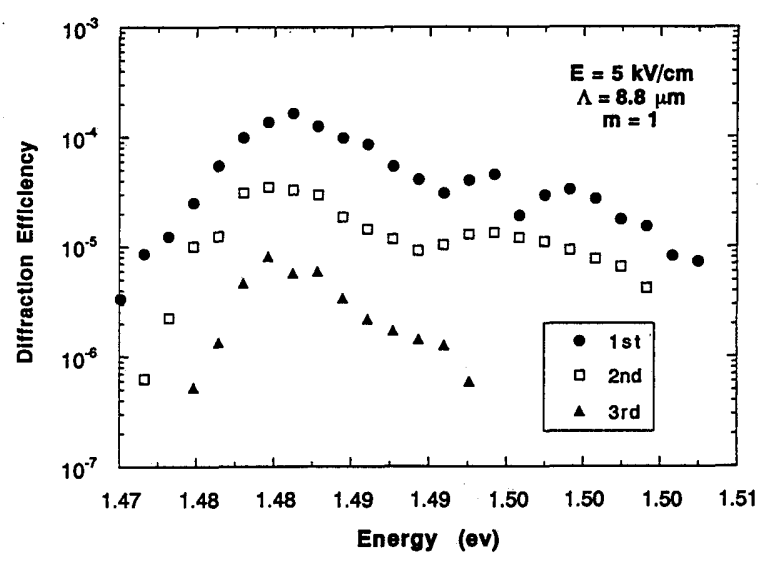

Fig. 12. Diffraction efficiencies for the first, second, and third spatial harmonic gratings as functions of photon energy for $m=1$. 


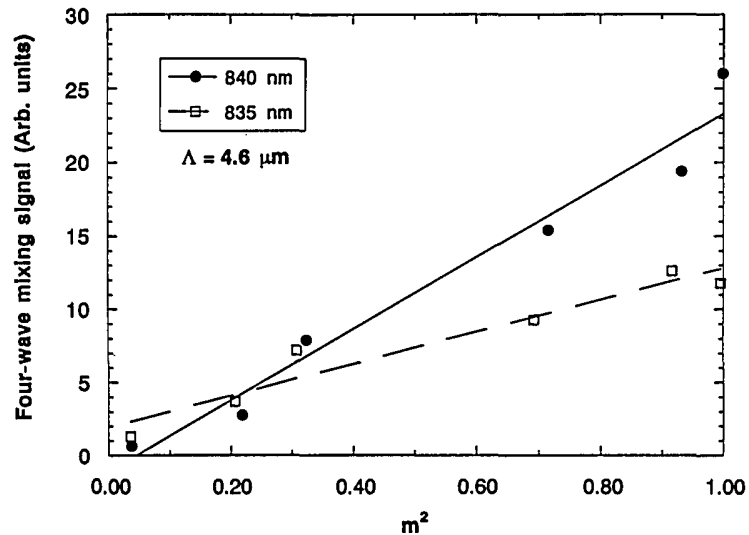

Fig. 13. Diffraction signal from the first-harmonic grating versus the square of the modulation index. The dependence is approximately linear, even for $m$ close to 1 .

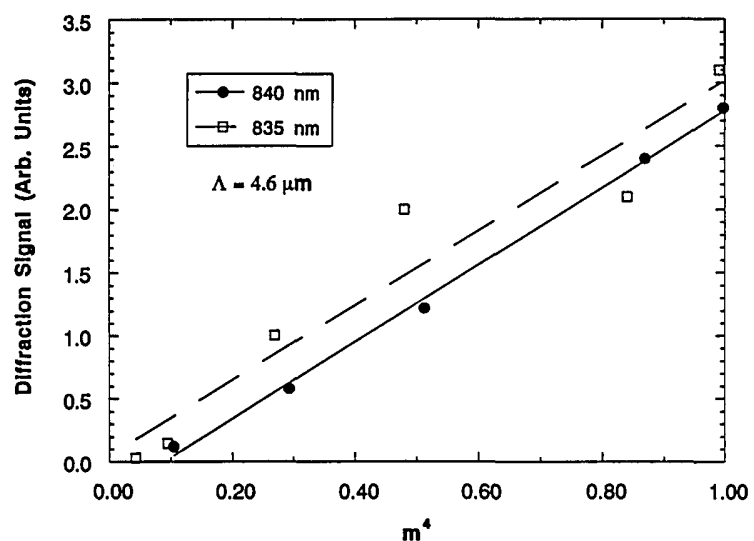

Fig. 14. Diffraction signal from the second-harmonic grating versus the fourth power of the modulation index. The dependence is approximately linear even for $m$ close to 1 .

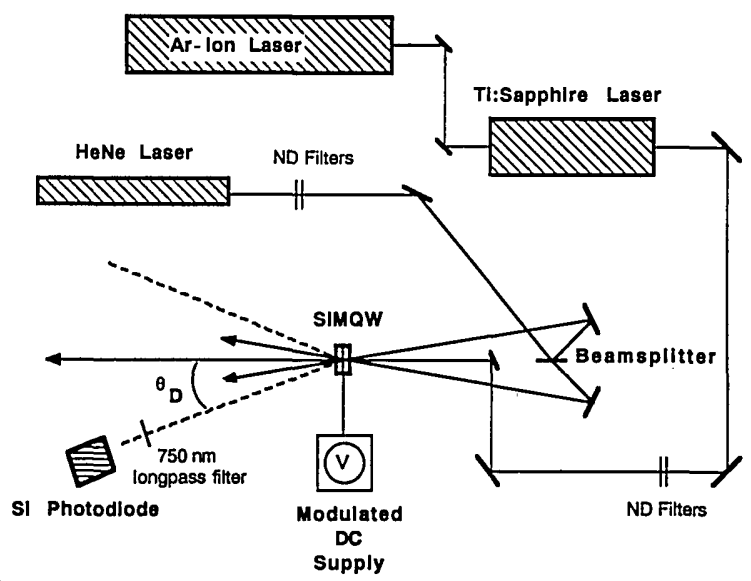

Fig. 15. Experimental geometry of nondegenerate four-wave mixing. The gratings are generated by a He-Ne laser and are probed by an infrared beam that is tuned through the exciton absorption. SIMQW, semi-insulating multiple quantum well; $\mathrm{ND}$, neutral-density.

Figs. 13 and 14. The diffraction efficiencies exhibit an approximately $m^{2}$ and $m^{4}$ dependence at both wavelengths, which is consistent with expectation and also in agreement with earlier experimental and numerical results. ${ }^{38,39}$ The second-harmonic grating can also be observed in a nondegenerate geometry. However, the effect is much weaker than in the degenerate case.

\section{Nondegenerate Four-Wave Mixing}

The mixing geometry for nondegenerate four-wave mixing is shown in Fig. 15. The gratings are written by a $\mathrm{He}-\mathrm{Ne}$ laser beam that is split by a beam splitter into two coherent beams that intersect at the semi-insulating multiple-quantum-well sample. We are able to use an above-band-gap laser because of the thin sample size. In spite of the large absorption of the $\mathrm{He}-\mathrm{Ne}$ wavelength $\lambda=633 \mathrm{~nm}$, there is an appreciable intensity of the pump beams throughout the multiple-quantum-well region. This feature of the photorefractive quantum wells introduces the possibility of above-band-gap holography. ${ }^{40}$ The gratings are probed by an infrared laser that is tuned to the exciton wavelengths. The probe beam strikes the sample at normal incidence. The diffraction angle $\theta_{D}$ for first-order diffraction is given by

$$
\sin \theta_{D}=2 \frac{\lambda_{p}}{\lambda_{\mathrm{HeNe}}} \sin \theta_{\mathrm{HeNe}},
$$

where $\theta_{\mathrm{HeNe}}$ is the half-angle of the He-Ne pump beam and $\lambda_{p}$ is the wavelength of the infrared probe. The diffracted signal is detected by a Si photodiode locked into the frequency of the electric-field modulation.

The diffracted signal as a function of the infrared probe wavelength is shown in Fig. 16 for a fringe spacing of $2.2 \mu \mathrm{m}$, with an electric field of $4 \mathrm{kV} / \mathrm{cm}$. The positions of the heavy- and light-hole excitons are included in the figure. The data represent the experimentally measured diffraction efficiency. The solid curve is the fit of Eq. (4.13) based on the electroabsorption and electrorefraction data in Fig. 5. The only adjustable parameter in the predicted curve is the magnitude of the diffraction efficiency.

The electric-field dependence of nondegenerate fourwave mixing is shown in Fig. 17 for a fringe spacing of $\Lambda=15 \mu \mathrm{m}$. The diffraction efficiency increases as the fourth power of the applied field up to $4 \mathrm{kV} / \mathrm{cm}$, as is expected from Eq. (4.13). However, for higher fields there is a significant deviation from the ideal behavior. There is no corresponding change in the current-voltage curves or in the electroabsorption curves for these fields. Therefore this qualitative change in the diffraction at high

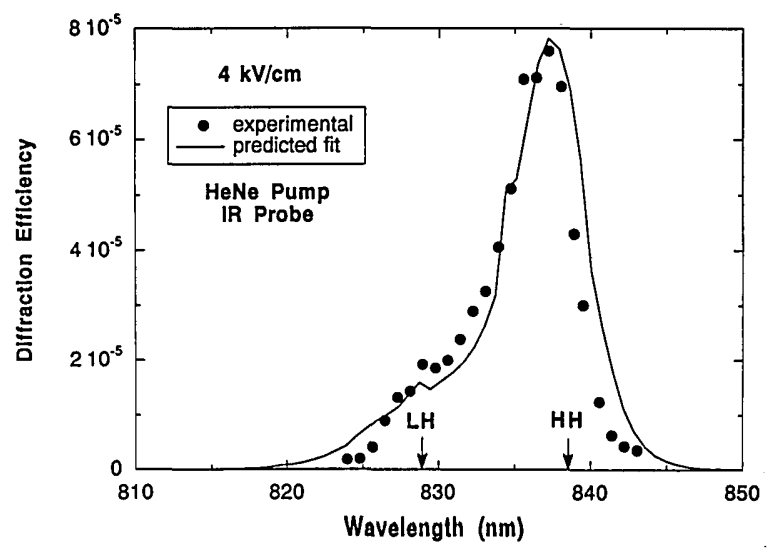

Fig. 16. Diffraction efficiency verus wavelength of the probe for an applied field of $4 \mathrm{kV} / \mathrm{cm}$ and $\Lambda=2.2 \mu \mathrm{m}$. The solid curve is fitted based on Fig. 5 . 


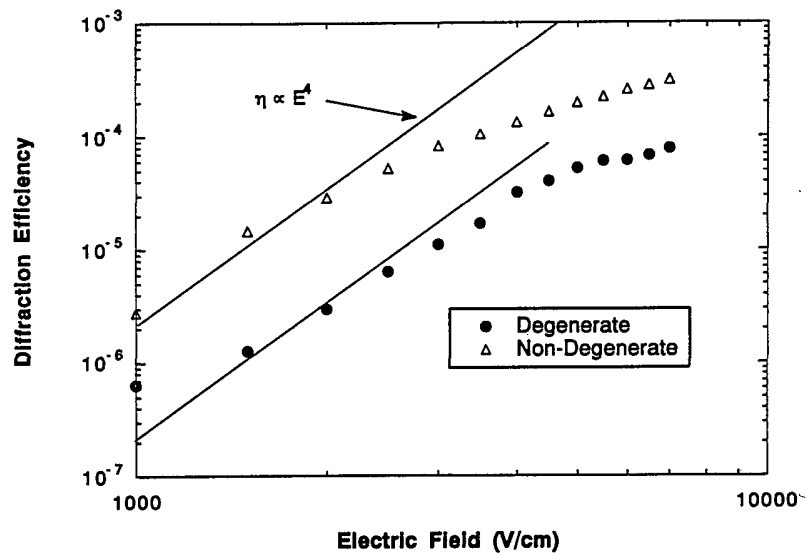

Fig. 17. Diffraction efficiency versus electric field for degenerate and nondegenerate four-wave mixing with $\Lambda=15 \mu \mathrm{m}$. Degenerate efficiencies are consistently smaller than nondegenerate efficiencies. At a low field, the diffraction efficiencies vary approximately with the fourth power of the applied field. Above $4 \mathrm{kV} / \mathrm{cm}$ there is a significant deviation from ideal behavior.

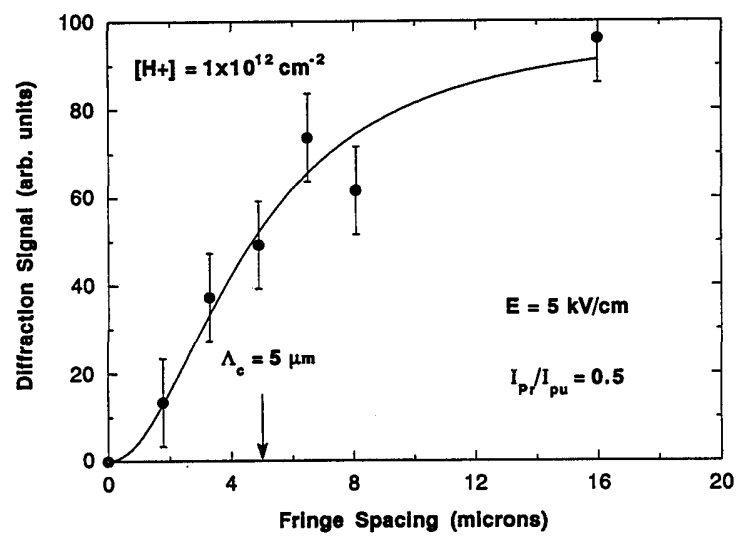

Fig. 18. Fringe-spacing dependence of the diffraction efficiency. The roll-off at small fringe spacings matches the behavior predicted in Fig. 7 based on diffusion lengths.

fields must be related to the processes that generate the photorefractive gratings within the quantum wells. One possibility is space-charge limitation. However, the results of computer simulations based on Eq. (3.12) make this possibility unlikely. It is also unlikely because of the estimated defect density of $10^{17} \mathrm{~cm}^{-3}$ produced by the proton implantation. An alternative possibility is hot carriers. Fields near $5 \mathrm{kV} / \mathrm{cm}$ are sufficient to heat carriers several hundred degrees above the lattice temperature. At these temperatures vertical transport out of the wells becomes strong. Carrier diffusion within the wells also becomes strong, which can have a large effect on the space-charge field, as shown in Eq. (3.16). Understanding this deviation will require more study. It is intriguing to note that this deviation occurs at the same electric field strengths for which the phase shift in two-wave mixing approaches $90^{\circ}$.

The fringe-spacing dependence of the diffracted signal is shown in Fig. 18 for a field of $5 \mathrm{kV} / \mathrm{cm}$. Included in the figure is a fit from the solution of Eq. (3.16). From the figure, the characteristic length $\Lambda_{c}$ is $5 \mu \mathrm{m}$, corresponding to a diffusion length of $L_{D}=0.8 \mu \mathrm{m}$ obtained from Eq. (3.17). The $\mu \tau$ product for this diffusion length, given in Eq. (3.18), is $\mu \tau=2 \times 10^{-7} \mathrm{~cm}^{2} / \mathrm{Vs}$. This value is con- sistent with the value $\mu \tau=3 \times 10^{-7} \mathrm{~cm}^{2} / \mathrm{Vs}$ obtained from photoconductivity measurements in Section 2 .

The photorefractive quantum-well structures represent a unique situation for photorefractive materials. In nondegenerate mixing the pump and the probe beams are absorbed in different portions of the structure. Therefore the space charge stored in the AlGaAs barriers does not erase with increasing probe intensity until sufficient carrier density is developed in the GaAs wells to overcome the barriers. This feature should lead to novel effects that have no analog in bulk materials. One of these possible effects is robust gratings. ${ }^{40}$ If the space charge in the barriers is isolated from the photoconductivity of the probe laser, then the gratings should persist up to high probe intensities. In spite of this effect, the space charge generated in the wells should be able to screen the spacecharge fields of the barriers.

To test these possibilities, we have studied the intensity dependence of the diffracted signal in nondegenerate fourwave mixing. The specific point being tested is the possibility of space-charge limitation, too few traps in the wells to screen the space-charge trapped in the barriers. If there are insufficient traps in the wells, than at smaller fringe spacings the gratings should persist up to higher probe intensities. The dependence of the diffraction signal on the probe intensity is shown in Fig. 19 for four fringe spacings, varying from 3.2 to $14.1 \mu \mathrm{m}$. No shift of the diffraction peak was observed. Further to test the possibility of explicitly observing the effects of spacecharge trapped in the barriers, we compared sample QW-2 with RB-1. In sample RB-1 the barriers are $150 \AA$ wide, compared with $100 \AA$ for QW-1. With the wider barriers, a greater space charge can be trapped, which requires higher screening densities within the wells. The data are shown in Fig. 20. No significant difference is observed between the two samples.

Based on these experiments, we conclude that no explicit evidence exists for effects caused by the presence of space charge trapped in the barriers. However, this conclusion cannot explain significant differences between the diffraction efficiencies of degenerate, compared with nondegenerate, four-wave mixing. Nor have we been able to establish precise laser intensities for which the probe laser erases the $\mathrm{He}-\mathrm{Ne}$ gratings. Therefore no conclusions can

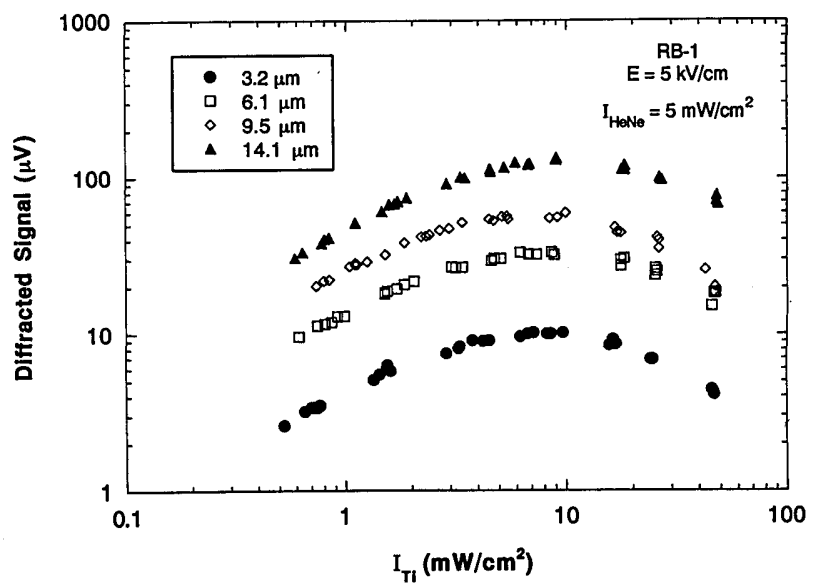

Fig. 19. Diffracted signal versus probe intensity for varying fringe spacings for sample RB-1 for an applied field of $5 \mathrm{kV} / \mathrm{cm}$. 


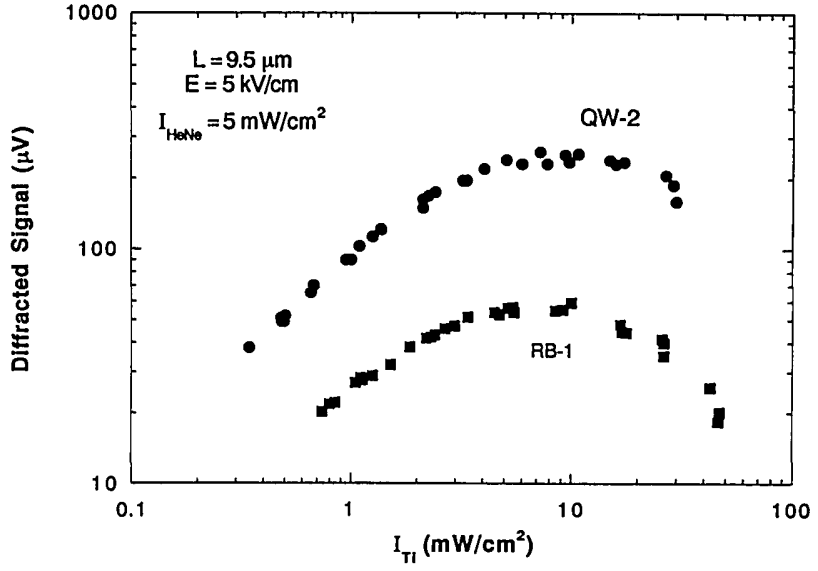

Fig. 20. Diffracted signal versus probe intensity for varying fringe spacings for samples QW-2 and RB-1 for $5 \mathrm{kV} / \mathrm{cm}$ and $\Lambda=9.5 \mu \mathrm{m}$.

be made at this time concerning the possible influence of the space charge trapped in the barriers on photorefractive properties. More study is needed to resolve this issue.

\section{TWO-WAVE MIXING}

\section{A. Two-Wave Coupling in a Thin Grating}

In two-wave mixing two coherent beams, $I_{1}$ and $I_{2}$, exchange energy. The intensity in the direction of $I_{1}$ is given by ${ }^{41}$

$$
\begin{aligned}
I_{1}{ }^{+}= & I_{1}\left(1-\bar{\alpha}_{E} L\right) \\
& +\left(I_{1} I_{2}\right)^{1 / 2}\left(+\frac{\Delta \alpha L \cos \phi}{2 \cos \theta^{\prime}}+\frac{2 \pi \Delta n L \sin \phi}{\lambda \cos \theta^{\prime}}\right),
\end{aligned}
$$

where $\bar{\alpha}_{E}$ represents the effect of the average square-field change on the absorption coefficient of the thin layer. In the direction of propagation of $\mathrm{I}_{2}$, we have similarly

$$
\begin{aligned}
I_{2}{ }^{+}= & I_{2}\left(1-\bar{\alpha}_{E} L\right) \\
& +\left(I_{1} I_{2}\right)^{1 / 2}\left(+\frac{\Delta \alpha L \cos \phi}{2 \cos \theta^{\prime}}-\frac{2 \pi \Delta n L \sin \phi}{\lambda \cos \theta^{\prime}}\right) .
\end{aligned}
$$

According to Eqs. (5.1) and (5.2), both the electroabsorption and the refractive-index grating contribute to the beam-coupling effect. However, the refractive-index grating causes an asymmetric effect with respect to the phase shift $\phi$ (controlled by the direction of the applied field), while the electroabsorption causes a symmetric effect.

\section{B. Two-Wave Mixing}

Two-wave mixing measurements were performed on the semi-insulating multiple quantum wells. The intensity modulation of a signal beam was measured while a pump beam was mechanically chopped at a frequency of $290 \mathrm{~Hz}$. A dc field was applied across the sample. The observed intensity modulation is composed of the effects from both electroabsorption and electrorefraction. By taking advantage of the quadratic quantum-confined exciton electroabsorption, the contributions of the symmetric and asymmetric parts of Eqs. (5.1) and (5.2) can be separated. The phase shift $\phi$ changes sign when the direction of the applied field is reversed. An effective electroabsorption coefficient $\Delta \alpha_{\text {eff }}$, which combines the effect of the terms in the first and second sets of square brackets in Eq. (4.15), is defined by

$$
\frac{1}{2} \frac{I_{1}^{+}(E)+I_{1}^{+}(-E)}{I_{1}}=1-\Delta \alpha_{\text {eff }} \frac{L}{\cos \theta^{\prime}} .
$$

The effect of the energy exchange is given by

$$
\gamma=\frac{1}{2} \frac{I_{1}{ }^{+}(E)-I_{1}{ }^{+}(-E)}{I_{1}}=\left(\frac{I_{2}}{I_{1}}\right)^{1 / 2} \frac{2 \pi \Delta n L \sin \phi}{\lambda \cos \theta^{\prime}} .
$$

Because the transport in semi-insulating multiple quantum wells is similar to that in bulk materials, it is reasonable to assume that $E_{\mathrm{sc}}$ is proportional to the modulation index of light intensity $m$. Then $\Delta n$ is also proportional to $m$. Therefore the above equation can be expressed as

$$
\gamma=[\beta /(1+\beta)] \Gamma L,
$$

where

$$
\Gamma=\frac{4 \pi \Delta n_{m} \sin \phi}{\lambda \cos \theta^{\prime}}
$$

and $\Delta n_{m}$ is the amplitude of the refractive-index grating for $m=1$ and $\beta=I_{2} / I_{1}$ is the beam intensity ratio.

The effective electroabsorption coefficient $\Delta \alpha_{\text {eff }}$ that is due to the mixing is plotted in Fig. 21, compared with the incoherent differential transmission measured with a single beam for an external $0-5-\mathrm{kV} / \mathrm{cm}$ ac field. Notice that the differential transmission and the effective electroabsorption have the same sign, which may apppear to be counterintuitive. This is because the $\Delta \alpha$ term in parentheses in Eq. (5.1), which represents diffraction by the absorption grating, wins out over the increase in absorption from the average electroabsorption $\bar{\alpha}_{E}$. Therefore there is a net decrease in the overall absorption because of the coherent interference between the two beams.

The gain $\Gamma$ is shown in Fig. 22 compared with the electrorefraction data derived from the Kramers-Kronig analysis of the incoherent differential transmission data in Fig. 4. The result is in good agreement with the prediction of Eqs. (5.1) and (5.2), giving unambiguous evidence for photorefractive gratings in this coupling process. No gain was observed when the writing beams were cross polarized with respect to each other. The electric-field dependence of the gain is shown in Fig. 23 for a fringe

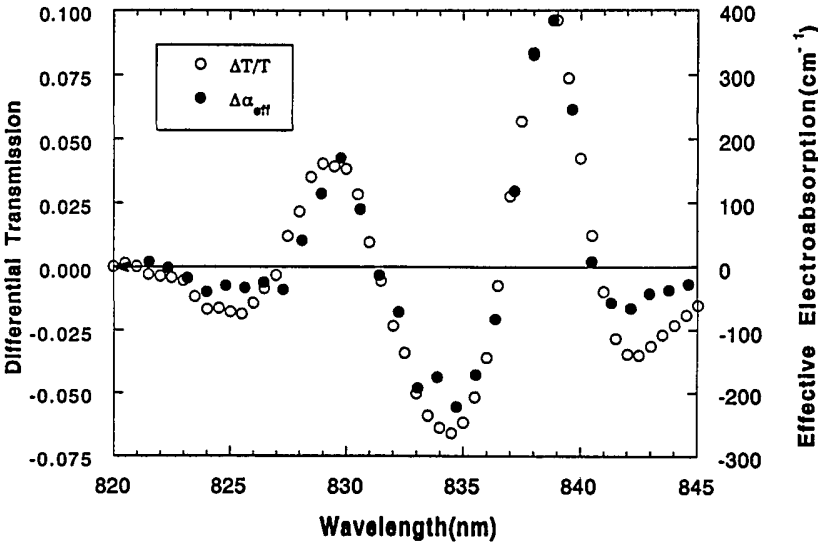

Fig. 21. Effective electroabsorption compared with differential transmission as functions of probe wavelength for an applied field of $7 \mathrm{kV} / \mathrm{cm}$ and a fringe spacing of $\Lambda=7.3 \mu \mathrm{m}$. 


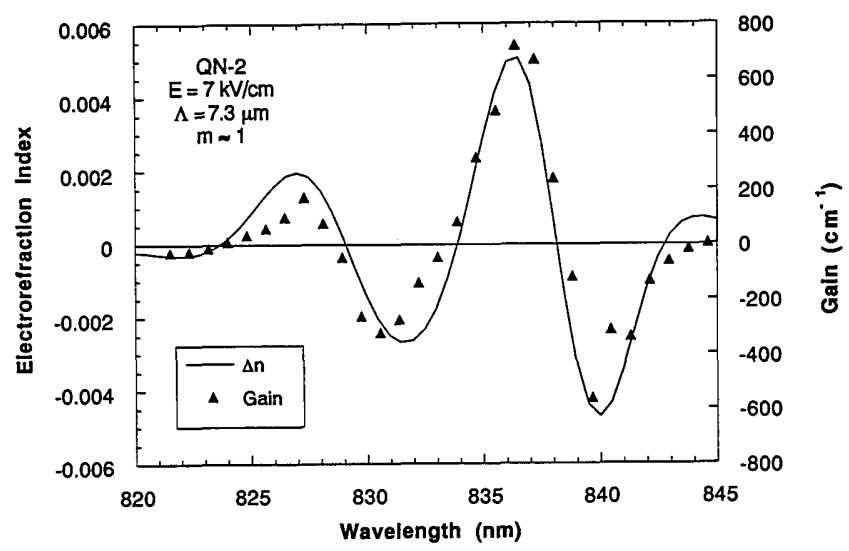

Fig. 22. Photorefractive gain compared with electrorefraction of sample QW-2 for $E=7 \mathrm{kV} / \mathrm{cm}, \Lambda=7.3 \mu \mathrm{m}$, and $m=1$.

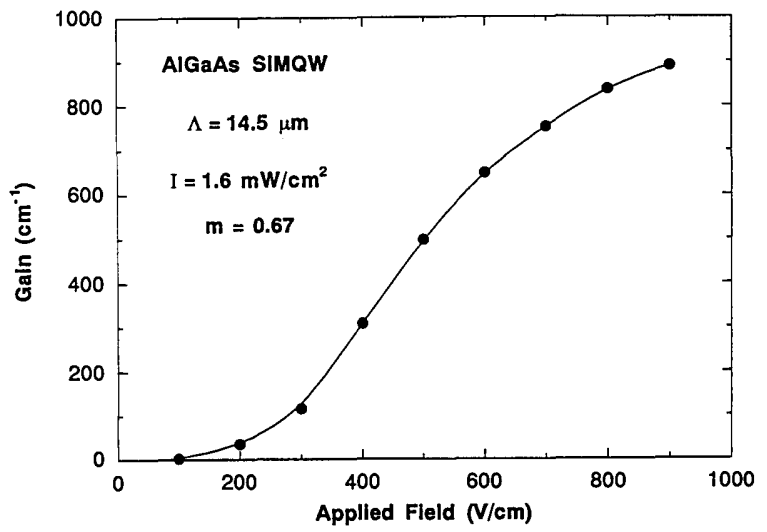

Fig. 23. Photorefractive gain as a function of applied field. The gain approaches $1000 \mathrm{~cm}^{-1}$ for a field of $10^{4} \mathrm{~V} / \mathrm{cm}$.

spacing of $14.5 \mu \mathrm{m}$ and a laser intensity of $1.6 \mathrm{~mW} / \mathrm{cm}^{2}$. The maximum gain obtained in our measurement approached $1000 \mathrm{~cm}^{-1}$ for $E=10^{4} \mathrm{~V} / \mathrm{cm}$. To our knowledge, this represents the largest photorefractive gain measured in any material. In our case, however the absorption losses still exceed the gain.

\section{Phase Shift}

The phase shift was determined by measuring two-wave mixing and degenerate four-wave mixing together at a zero in the electroabsorption. For two-wave mixing the relative intensity modulation is given by Eq. (5.4). This includes dependence on the phase shift $\phi$ and the index grating but not the absorption grating. At the same wavelength and fringe spacing the square root of the diffraction efficiency is

$$
\sqrt{\eta}=\pi \Delta n L /\left(\lambda \cos \theta^{\prime}\right)
$$

which depends only on the index grating, not on the phase shift. Therefore the phase shift can be isolated by using

$$
\sin \phi=\frac{1}{2 \sqrt{\beta}}\left(\frac{\Delta I / I}{\sqrt{\eta}}\right) .
$$

The dependence on the $E$ field for several different fringe spacings is shown in Fig. 24. The phase shift increases with the applied field, coming close to $\pi / 2$ for high fields.
In bulk photorefractive materials, for there to be a significant phase shift the applied field should be either much lower than the diffusion field $E_{D}$ or comparable with the maximum internal field modulation. The internal field modulation is limited by the space-charge density $N_{t}$ and the defect occupancy $f$ in the expression $E_{\max }=e f(1-$ $f) N_{t} /\left(K \epsilon \epsilon_{0}\right)$. For a conservative estimate of the defect density of $f(1-f) N_{t}=1 \times 10^{16} \mathrm{~cm}^{-3}$ and a $5-\mu \mathrm{m}$ fringe spacing, $E_{\max }=1 \times 10^{5} \mathrm{~V} / \mathrm{cm}$, compared with our maximum field $E_{0}=1 \times 10^{4} \mathrm{~V} / \mathrm{cm}$. We have performed computer simulations for bulk photorefractive properties, using parameters identical to the experimental conditions, and find no significant phase shift. Therefore the phase shift is expected to arise from vertical transport of carriers out of the quantum wells and into or over the barriers. More research is needed to resolve the issue of the phase shift.

\section{ULTRAHIGH-SENSITIVITY OPTICAL NONLINEARITY}

Photorefractive quantum-well materials are ideally suited for operation under extremely low light powers. The high resistivity of the semi-insulating samples, coupled with the large absorption coefficient for electron-hole generation, leads to low saturation intensities. In addition, the large electro-optic effects of quantum-confined excitons yield large interaction strengths. Combining the large interaction strengths with the low saturation intensities leads to one of the highest-sensitivity optical nonlinearities measured in any material system. In this section two-wave mixing is used to demonstrate the low-intensity operation of the quantum wells. Two-wave mixing has an advantage over four-wave mixing in that for two-wave mixing relatively small changes in the refractive index $\Delta n$ can be measured with good sensitivity. This is because the energy transfer between the mixing beams is linearly proportional to $\Delta n$, as opposed to the square of this value for four-wave mixing.

\section{A. Saturation Intensity}

Saturation is perhaps one of the simplest optical nonlinearities. In the photorefractive effect, saturation occurs when the photoconductivity exceeds the dark conductivity. The generation of the refractive index and the absorption

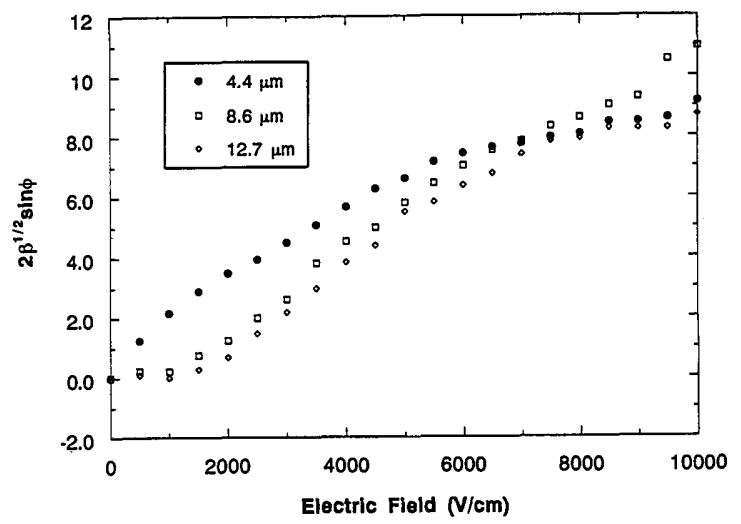

Fig. 24. Sine of the phase shift as a function of electric field for varying fringe spacings. Above $4 \mathrm{kV} / \mathrm{cm}$ the phase shift is close to $\pi / 2$, with which $\beta \approx 25$ is ideal for maximum photorefractive gain. 


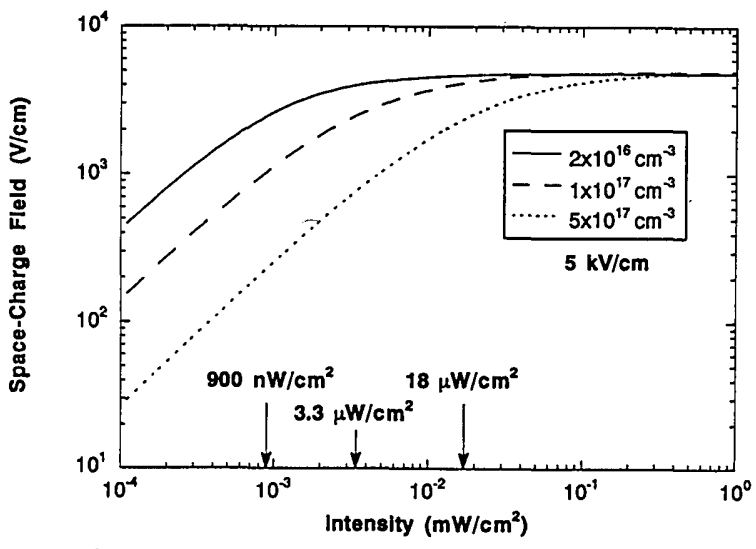

Fig. 25. Simulation of space-charge field amplitude as a function of laser intensity for various defect densities.

gratings created by the spatially modulated intensity $I(x)$ is balanced against erasure of the space-charge gratings by dielectric relaxation from the background conductivity. The condition for intensity saturation is

$$
I_{\text {sat }}=n_{d} / \alpha \tau,
$$

where $n_{d}$ is the dark carrier density and the lifetime $\tau$ is the larger of $\tau_{e}$ and $\tau_{h}$. The dark density $n_{d}$ is set by the position of the Fermi level in the band gap. In semiinsulating materials the Fermi level is near midgap, producing small values of dark carrier densities. Small values of $n_{d}$ and the large value of $\alpha$ produce a low saturation intensity. A larger lifetime will also favor a lower saturation intensity. The lifetime $\tau$ is inversely proportional to the defect density, so lower defect densities will give lower saturation intensities. However, if the defect density is too low, then the Fermi level will no longer be pinned midgap, so that higher saturation densities will be produced. A longer lifetime will also yield a longer diffusion length and a degradation of device resolution. There are clearly trade-offs among the properties of saturation intensity, device speed, and device resolution, based on the choice of the appropriate defect density for a given application.

The solution of Eq. (3.12) for three different defect densities is shown in Fig. 25 for low intensities and the parameters in Table 2. The magnitude of the space-charge field increases linearly with increasing intensity below the saturation intensity and saturates for higher intensities. In the simulations the Fermi level is taken to be the intrinsic Fermi level of GaAs. The saturation intensities in this case are shown in Fig. 25 and vary between 1 and $20 \mu \mathrm{W} / \mathrm{cm}^{2}$ for the defect densities chosen. The space charge can be expressed as

$$
E_{\mathrm{sc}}=m \zeta E_{0} I /\left(I_{\mathrm{sat}}+I\right),
$$

where $\zeta$ contains the features of carrier dynamics and electron-hole competition and the shift of $E_{\mathrm{sc}}$ relative to the intensity grating. The diffusion field has been neglected in the expression, although diffusion continues to be important for the transport processes contained in $\zeta$.

The intensity dependence of the two-wave mixing was measured for sample QW-2. By tuning the pump-laser wavelengths, the effects of index or absorption gratings were isolated from each other. Absorption gratings are associated with beam modulation that is symmetric with respect to both beams. Index gratings are associated with beam modulation that is asymmetric with respect to both beams. The asymmetric signal is photorefractive beam coupling in which energy is transferred from one beam to the other. The beam modulations caused by an index grating and by an absorption grating are shown in Fig. 26 for $\lambda=836 \mathrm{~nm}$ and $\lambda=839 \mathrm{~nm}$, respectively, as functions of laser intensity for $m=0.67$. The first wavelength corresponds to a zero crossing in the electroabsorption, and the second corresponds to a zero crossing in the electrorefraction of Fig. 4. The differences in the amplitudes depend on differences between $s_{1}$ and $s_{2}$ in Fig. 5 for the two different wavelengths. Both modulation signals vary linearly with laser intensity below the saturation intensity and saturate to constant values above the saturation intensity. The best fits to Eq. (6.2) are shown, yielding saturation intensities of 10 and $22 \mu \mathrm{W} / \mathrm{cm}^{2}$ for the index and absorption gratings, respectively. The smaller saturation value for the index grating is caused by a larger absorption coefficient at $\lambda=836 \mathrm{~nm}$. The saturation intensities observed in Fig. 26 are consisitent with the intensities predicted by the simulations in Fig. 25. The simulations assumed that the Fermi level was positioned at the intrinsic level in the band gap. These saturation measurements therefore demonstrate that the Fermi level in proton-implanted quantum wells lies close to the intrinsic value.

\section{B. Nonlinear Optical Sensitivity}

The refractive-index and absorption grating magnitudes increase linearly with laser intensity for $I<I_{\text {sat }}$. For this range of intensities the photorefractive effect can be regarded as an $n_{2}$ or an $\alpha_{2}$ process, defined by

$$
n=n_{0}+n_{2} I, \quad \alpha=\alpha_{0}+\alpha_{2} I .
$$

Experimental values of $n_{2}$ and $\alpha_{2}$ are obtained by dividing the measured index and absorption grating magnitudes $\Delta n$ and $\Delta \alpha$ by $I_{\text {sat }}$ :

$$
n_{2}=\Delta n / I_{\text {sat }}, \quad \alpha_{2}=\Delta \alpha / I_{\text {sat }} .
$$

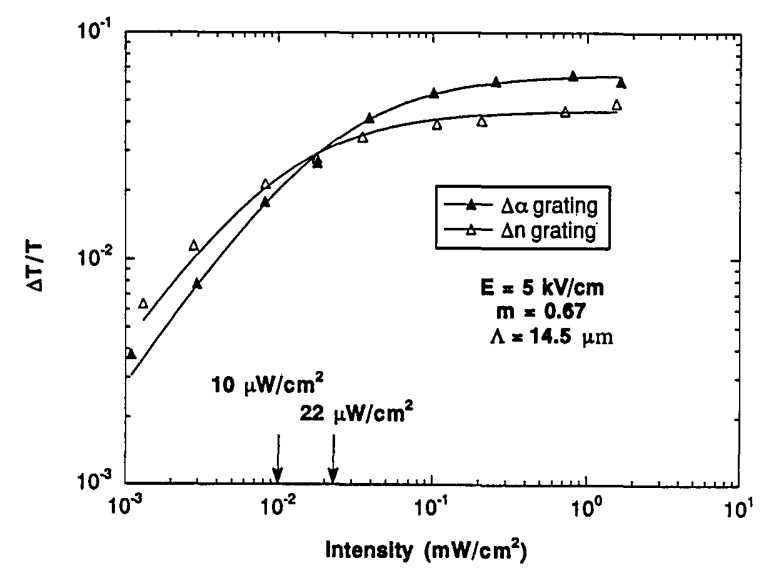

Fig. 26. Experimental values of modulated intensity in twowave mixing as a function of laser intensity for $E=5 \mathrm{kV} / \mathrm{cm}$. The absorption grating data were obtained for $\lambda=839 \mathrm{~nm}$, and the refractive-index grating data were obtained for $\lambda=836 \mathrm{~nm}$. Saturation intensities in the range of $10 \mu \mathrm{W} / \mathrm{cm}^{2}$ are observed. 
In our experiments we applied electric fields as large as $10 \mathrm{kV} / \mathrm{cm}$. With these fields the observed grating amplitudes were

$$
\Delta n=0.007, \quad \Delta \alpha=1400 \mathrm{~cm}^{-1}
$$

at 836 and $839 \mathrm{~nm}$, respectively. The corresponding nonlinear coefficients are

$$
n_{2}=7 \times 10^{2} \mathrm{~cm}^{2} / \mathrm{W}, \quad \alpha_{2} / \alpha_{0}=9 \times 10^{3} \mathrm{~cm}^{2} / \mathrm{W} .
$$

This third-order optical nonlinearity is one of the largest optical nonlinearities generated to date. The physical processes of photorefractive quantum wells are similar to those of self-electro-optic devices, ${ }^{42}$ and our value of $n_{2}$ compares favorably with values of $n_{2} \approx 5 \times 10^{2} \mathrm{~cm}^{2} / \mathrm{W}$ obtained from incoherent optical nonlinearities in selfelectro-optic devices. However, in our case the nonlinear optical mixing is based on the mutual coherence of the exciting lasers and therefore provides the basis for diffraction-limited holographic applications.

\section{ACKNOWLEDGMENTS}

M. R. Melloch acknowledges support by U.S. Office of Naval Research grant N00014-89-J-1864. D. D. Nolte acknowledges support by National Science Foundation grant ECS-9008266, the Presidential Young Investigator Progam, and the Alfred P. Sloan Foundation.

\section{REFERENCES}

1. A. M. Glass, A. M. Johnson, D. H. Olson, W. Simpson, and A. A. Ballman, Appl. Phys. Lett. 44, 948 (1984).

2. A. M. Glass and J. Strait, in Photorefractive Materials and Their Applications I, P. Günter and J.-P. Huignard, eds. (Springer-Verlag, Berlin, 1988), p. 237.

3. V. W. Franz, Z. Naturforsch. A 13, 484 (1958).

4. L. V. Keldysh, Sov. Phys. JETP 34, 788 (1958).

5. J. Callaway, Phys. Rev. 130, 549 (1963).

6. K. Thermalingham, Phys. Rev. 130, 2204 (1963).

7. J. Callaway, Phys. Rev. 134, A998 (1964).

8. H. D. Rees, Solid State Commun. 5, 365 (1967).

9. J. D. Dow and D. Redfield, Phys. Rev. B 1, 3358 (1970).

10. F. Stern, Phys. Rev. 133, A1653 (1964).

11. B. O. Seraphin and N. Bottka, Phys. Rev. 139A, 560 (1965).

12. T. E. Van Eck, L. M. Walpita, W. S. C. Chang, and H. H. Wieder, Appl. Phys. Lett. 48, 451 (1986).

13. A. Alping and L. A. Coldren, J. Appl. Phys. 61, 2430 (1987).

14. J. E. Zucker, T. L. Hendrickson, and C. A. Burrus, Appl. Phys. Lett. 52, 946 (1988).

15. J. E. Millerd, S. D. Koehler, E. M. Garmire, A. Partovi, A. M. Glass, and M. B. Klein, Appl. Phys. Lett. 57, 2776 (1990).
16. A. Partovi, A. Kost, E. M. Garmire, G. C. Valley, and M. B. Klein, Appl. Phys. Lett. 56, 1089 (1990).

17. D. S. Chemla, D. A. B. Miller, P. W. Smith, A. C. Gossard, and W. Wiegmann, IEEE J. Quantum Electron. QE-20, 265 (1984).

18. D. D. Nolte, D. H. Olson, G. E. Doran, W. H. Knox and A. M. Glass, J. Opt. Soc. Am. B 7, 2217 (1990).

19. E. Yablonovitch, T. Gmitter, J. P. Harbison, and R. Bhat, Appl. Phys. Lett. 51, 2222 (1987).

20. Y. Silverberg, P. W. Smith, D. A. B. Miller, B. Tell, A. C. Gossard, and W. Wiegmann, Appl. Phys. Lett. 46, 701 (1985).

21. M. B. Johnson, T. C. McGill, and N. G. Paulter, Appl. Phys. Lett. 54, 2424 (1989).

22. H. J. Stein, Appl. Phys. Lett. 57, 792 (1990).

23. M. Lambsdorff, J. Kuhl, J. Rosenzweig, A. Axmann, and J. Schneider, Appl. Phys. Lett. 58, 1881 (1991).

24. M. Schultz and H. Weiss, in Landolt-Börnstein Numerical Data and Functional Relationships in Science and Technology, K:-H. Hellwege and A. M. Hellwege, eds. (SpringerVerlag, Berlin, 1984), Vol. 17d, p. 97.

25. B. Schwartz, L. A. Koszi, P. J. Anthony, and R. L. Hartman, J. Electrochem. Soc. 131, 1703 (1984).

26. K. Kenefick, J. Electrochem. Soc. Solid-State Sci. Technol. 129, 2380 (1982).

27. K. J. K. C. Juang and R. B. Darling, J. Vac. Sci. Technol. B 8, 1122 (1990).

28. E. Yablonovitch, D. M. Hwang, T. J. Gmitter, L. T. Florez, and J. P. Harbison, Appl. Phys. Lett. 56, 2419 (1990).

29. K. Seeger, Semiconductor Physics (Springer-Verlag, Berlin, 1985).

30. S. M. Sze, Physics of Semiconductor Devices (New York, Wiley-Interscience, 1969).

31. D. A. B. Miller, D. S. Chemla, T. C. Damen, A. C. Gossard, W. Wiegmann, T. H. Wood, and C. A. Burrus, Phys. Rev. Lett. 53, 2173 (1984).

32. F. L. Lederman and J. D. Dow, Phys. Rev. B 13, 1633 (1976).

33. D. A. B. Miller, D. S. Chemla, T. C. Damen, A. C. Gossard, W. Wiegmann, T. H. Wood, and C. A. Burrus, Phys. Rev. B 32, 1043 (1985).

34. D. A. B. Miller, D. S. Chemla, and S. Schmitt-Rink, Phys. Rev. B 33, 6976 (1986).

35. G. C. Valley and J. F. Lam, in Photorefractive materials and Their Applications I, P. Günter and J.-P. Huignard, eds. (Springer-Verlag, Berlin, 1988), p. 75.

36. M. G. Moharam, T. K. Gaylord, and R. Magnusson, Opt. Commun. 32, 19 (1980).

37. Q. N. Wang, D. D. Nolte, and M. R. Melloch, Opt. Lett. 16, 1944 (1991).

38. M. P. Petrov, S. V. Miridonov, S. I. Stepanov, and V. V. Kulikov, Opt. Commun. 31, 301 (1979).

39. F. Vachss and L. Hesselink, J. Opt. Soc. Am. A 5, 690 (1988).

40. D. D. Nolte, Q. N. Wang, and M. R. Melloch, Appl. Phys. Lett. 58, 2067 (1991).

41. Q. N. Wang, D. D. Nolte, and M. R. Melloch, Appl. Phys. Lett. 59, 256 (1991).

42. D. A. B. Miller, D. S. Chemla, T. C. Damen, T. H. Wood, C. A. Burrus, A. C. Gossard and W. Wiegmann, IEEE J. Quantum Electron. QE-21, 1462 (1985). 